$32^{n d}$ AIAA Applied Aerodynamics Conference, 16-20 June 2014, Atlanta, Georgia

\title{
Time-Spectral Rotorcraft Simulations on Overset Grids
}

\author{
Joshua I. Leffell* \\ Scott M. Murman ${ }^{\dagger}$ \\ Stanford University, Stanford, CA 94305 \\ NASA Ames Research Center, Moffett Field, CA 94035 \\ Thomas H. Pulliam $\ddagger$ \\ NASA Ames Research Center, Moffett Field, CA 94035
}

\begin{abstract}
The Time-Spectral method is derived as a Fourier collocation scheme and applied to NASA's overset Reynolds-averaged Navier-Stokes (RANS) solver OVERFLOW. The paper outlines the Time-Spectral OVERFLOW implementation. Successful low-speed laminar plunging NACA 0012 airfoil simulations demonstrate the capability of the Time-Spectral method to resolve the highly-vortical wakes typical of more expensive three-dimensional rotorcraft configurations. Dealiasing, in the form of spectral vanishing viscosity (SVV), facilitates the convergence of Time-Spectral calculations of high-frequency flows. Finally, simulations of the isolated V-22 Osprey tiltrotor for both hover and forward (edgewise) flight validate the three-dimensional Time-Spectral OVERFLOW implementation. The Time-Spectral hover simulation matches the time-accurate calculation using a single harmonic. Significantly more temporal modes and SVV are required to accurately compute the forward flight case because of its more active, high-frequency wake.
\end{abstract}

\section{Introduction}

C $\mathrm{F}$ chinery, and flapping wing configurations. The standard procedure for simulating such flows involves advancing the unsteady governing equations forward in time long enough for the initial transient to exit the computational domain and for a statistically stationary flow to be achieved. It is often necessary to simulate through several periods of motion to accomplish this task, making unsteady design optimization prohibitively expensive for many realistic problems. An effort to reduce the computational cost of these calculations led to the development of the Harmonic Balance method [1,2], which capitalizes on the periodic nature of the solution, while maintaing the ability to resolve nonlinearities inherent in the underlying physics. This approach exploits the fact that time-periodic flow, while varying in the time domain, is invariant in the frequency domain. Expanding the temporal variation at each spatial node as a Fourier series transforms the unsteady governing equations into a coupled set of steady equations in integer harmonics that can be tackled with the acceleration techniques afforded to steady-state flow solvers. Other similar approaches, such as the Nonlinear Frequency Domain (NLFD) $[3,4,5]$, Reduced Frequency [6], and Time-Spectral $[7,8,9]$ methods, were developed shortly thereafter and are examples of Fourier pseudospectral schemes.

Fourier pseudospectral methods have demonstrated marked success in reducing the computational costs associated with simulating forced periodic flows [4]. Using this approach, the solutions at $N$ equispaced time instances are coupled through the temporal derivative term. The trigonometric representation of periodic phenomenon provides spectral convergence as the number of time-samples, and correspondingly, the number of resolvable harmonics, $K=(N-1) / 2$, increases. The spectral convergence rate of Fourier methods is superior to the algebraic convergence rates associated with traditional time-marching schemes for unsteady calculations, implying that a given level of accuracy can be achieved with significantly fewer degrees of freedom [10]. Some approaches iterate the equations in the frequency domain directly. Others performs all operations in the time domain, simplifying the process of augmenting this capability to existing solvers. However, each approach harnesses the underlying steady solution in the frequency domain.

\footnotetext{
*Department of Aeronautics \& Astronautics. AIAA Student Member. jleffell@stanford.edu

${ }^{\dagger}$ AIAA Member. Scott.M.Murman@nasa.gov

¥AIAA Associate Fellow. Thomas.H.Pulliam@nasa.gov

This material is declared a work of the U.S. Government and is not subject to copyright protection in the United States.
} 
Overset and Cartesian grid methodologies are versatile techniques capable of handling complex-geometry configurations with relative motion between components, and are commonly used for practical engineering applications. The combination of the Time-Spectral approach with this general capability may potentially provide an enabling new design and analysis tool. In an arbitrary moving-body scenario for these approaches, a Lagrangian body moves through a fixed Eulerian mesh (or another moving Lagrangian mesh). Mesh points interior to solid bodies are removed (cut or blanked), leaving a hole in the background mesh. Blanked mesh points are excluded from the computational domain within which the governing equations are solved, and therefore the solution at such nodes is undefined. In general, such grid points undergo dynamic blanking where they are blanked only for a fraction of time and active otherwise. Dynamically-blanked nodes lack a complete set of time samples, preventing direct application of the Time-Spectral approach due to the infinite support of the complex exponential basis functions of the Fourier series upon which the method is based. Murman [6] demonstrated the approach for a Cartesian solver with rigid domain motion, wherein the hole cutting remained fixed. Similarly, Thomas et al. [11,12] and Custer [13] applied the method to the NASA overset OVERFLOW solver with static hole-cutting. Soucy et al. [14] avoided dynamically-blanked nodes by extending the near-body grids such that they envelop the solid body at all time instances. This approach proves worthy for simple configurations but is not a general solution for the case of arbitrary motion. These efforts focused on applying this method to Cartesian and overset meshes with constant blanking. Mavriplis et al. [15] demonstrated an approach to treat dynamically-blanked nodes by applying a Laplacian smoothing operator to populate blanked data from surrounding grid points. Recently, the authors developed a hybrid Time-Spectral scheme implemented within NASA's OVERFLOW solver to consistently handle dynamicallyblanked nodes for overset relative-motion cases [16].

This paper extends the algorithm development from [16] by applying it to realistic three-dimensional rotorcraft problems. Representative experimental and computational flowfields of rotorcraft in forward flight are depicted in Figure 1. These images demonstrate some of the complexities inherent in rotorcraft problems such as blade-vortex interaction (BVI) and the convection of highly dynamical vortices through a turbulent environment. Of particular interest are the high-frequency phenomenon associated with convecting vortices that are difficult to resolve, time-dependent features due the broad range of frequencies encountered.

Previous applications of Time-Spectral solvers to rotorcraft problems include the work of Tatossian and Nadarajah [17,18], Ekici et al. [19], Choi et al. [20,21] and Yang et al. [22]. Ekici et al. demonstrated the ability for the High Dimensional Harmonic Balance (HDHB) method to successfully calculate threedimensional solutions for the rotor of Caradonna and Tung [23] in hover and lifting and non-lifting forward flight. Tatossian and Nadarajah performed adjoint-based design optimization of the Caradonna and Tung rotor using an NLFD solver for both hover and forward flight. Choi et al. implemented exact fluid-structure coupling for Time-Spectral calculations and also performed adjoint-based aerodynamic shape optimization using a Time-Spectral solver for the UH-60A rotor. Yang et al. demonstrated the ability for an augmented $\mathrm{BDF} /$ Time-Spectral algorithm to resolve quasi-periodic maneuver for the UH-60A rotor.

The paper continues in $\S I I$ with a review of the Time-Spectral method followed by details of the OVERFLOW discretization in $\S I I I$. Numerical experiments in $\S I V$ include a series of low-speed laminar plunging airfoil cases that serve as informative model problems for the the three-dimensional rotorcraft cases. These simulations serve as more tractable two-dimensional cases that allow for more rigorous analysis to ascertain the solution procedures required for tackling the more expensive three-dimensional problems. Finally, in $\S \mathrm{V}$, realistic three-dimensional rotorcraft calculations for the isolated V-22 Tilt Rotor Aeroacoustic Model (TRAM) in hover and forward (edgewise) flight demonstrate the ability of the Time-Spectral scheme to resolve the flow.

\section{The Time-Spectral Method}

The standard Time-Spectral method is applicable to most PDE-based solution methods, but it cannot be directly incorporated into the class of solvers employing overset technology. Relative motion within the overset framework introduces spatial nodes that may not contain a physically meaningful solution for all time, due the the dynamic hole-cutting of points within a solid body. The solution at these nodes cannot be uniquely expanded in a basis of infinitely-supported complex exponential functions, and therefore these nodes can not be treated with the standard Time-Spectral approach. Our previous work [16] developed an approach to deal with such dynamically-blanked nodes by partitioning the temporal domain and expanding local expansions of the solution with a non-periodic basis derived from barycentric rational interpolants. 
The standard Time-Spectral method is derived as a Fourier collocation scheme [20,24] for a time-periodic solution, $u$, to a representative PDE, with a general residual operator, $\mathcal{R}(u)$, that is free to be of linear or nonlinear form.

$$
\frac{\partial}{\partial t} u(\mathbf{x}, t)+\mathcal{R}(u(\mathbf{x}, t))=0, \quad \mathbf{x} \in \Omega
$$

Since the solution is assumed a priori to be periodic in time, such that $u(\mathbf{x}, t+T)=u(\mathbf{x}, t)$, the solution at every point in space, $\mathbf{x}$, can be expressed as a Fourier series where the basis functions, $\phi_{k}(t)$, are the complex exponentials in integer harmonics of the fundamental frequency, $\omega=2 \pi / T$.

$$
u(\mathbf{x}, t)=\sum_{k=-\infty}^{\infty} \tilde{u}_{k}(\mathbf{x}) \phi_{k}(t), \quad \phi_{k}(t)=e^{i \omega k t}
$$

A discrete approximation, $u_{N}$, is required, and therefore the Fourier series is truncated to $N$ terms which retains $K=(N-1) / 2$ harmonics of the fundamental frequency.

$$
u_{N}(\mathbf{x}, t)=\sum_{k=-K}^{K} \tilde{u}_{k}(\mathbf{x}) \phi_{k}(t)
$$

The method of weighted residuals (MWR) is applied to approximate the solution to PDEs by minimizing a discrete residual, $R_{N}$, of Eq. 1 .

$$
R_{N}(\mathbf{x}, t)=\frac{\partial}{\partial t} u_{N}(\mathbf{x}, t)+\mathcal{R}\left(u_{N}(\mathbf{x}, t)\right), \quad \mathbf{x} \in \Omega
$$

It requires that $R_{N}$ integrates to zero against an appropriate set of test functions, $\psi$, over the period [25].

$$
\left(R_{N}, \psi_{j}\right)_{w}=\int_{0}^{T} R_{N} \psi_{j} w d t=0, \quad j \in J_{N}, \quad J_{N}=\{0, \ldots, N-1\}
$$

The choice of weight and test functions, $w=1$ and $\psi_{j}=\delta\left(t-t_{j}\right)$, respectively, defines a collocation scheme because it eliminates the discrete residual at each of the collocation points, $t_{j}$.

$$
\frac{\partial}{\partial t} u_{N}\left(\mathbf{x}, t_{j}\right)+\mathcal{R}\left(u_{N}\left(\mathbf{x}, t_{j}\right)\right)=0, \quad \mathbf{x} \in \Omega, \quad j \in J_{N}
$$

Because the temporal basis functions are the complex exponential functions, the collocation points are distributed uniformly over the period, $t_{j}=j T / N$.

The temporal derivative in Eq. 6 is evaluated by analytically differentiating the truncated Fourier series in Eq. 3.

$$
\frac{\partial}{\partial t} u_{N}(\mathbf{x}, t)=\sum_{k=-K}^{K} i \omega k \tilde{u}_{k}(\mathbf{x}) e^{i \omega k t}
$$

The Fourier coefficients, $\tilde{u}_{k}(\mathbf{x})$, are determined from the solution values at the $N$ collocation points by applying the discrete Fourier transform.

$$
\tilde{u}_{k}(\mathbf{x})=\frac{1}{N} \sum_{j=0}^{N-1} u_{N}\left(\mathbf{x}, t_{j}\right) e^{-i \omega k t_{j}}
$$

While this results in a pseudospectral method, it enables spatial operators from existing flow solvers to be leveraged because the spatial residual is evaluated in the time domain. Substitution of Eq. 8 into Eq. 7 defines the derivative at time $t_{j}$. The derivative at each collocation point is expressed as a weighted sum of the solution at every collocation point. This can be expressed in matrix form, where the time-domain temporal differentiation operator, $\mathcal{D}_{N}$, acts on the time-history of the solution, $\mathbf{u}_{N}(\mathbf{x})=\left\{u_{N}\left(\mathbf{x}, t_{0}\right), \ldots, u_{N}\left(\mathbf{x}, t_{N-1}\right)\right\}^{T}$.

$$
\frac{\partial}{\partial t} \mathbf{u}_{N}(\mathbf{x})=\mathcal{D}_{N} \mathbf{u}_{N}(\mathbf{x})
$$


Expressing Eq. 6 is matrix form and replacing the analytic temporal derivative with the operator in Eq. 9 results in the global system of equations.

$$
\mathcal{D}_{N} \mathbf{u}_{N}(\mathbf{x})+\mathcal{R}\left(\mathbf{u}_{N}(\mathbf{x})\right)=0, \quad \mathbf{x} \in \Omega
$$

To avoid directly solving a large or nonlinear system, Eq. 11 is iterated in pseudotime until satisfactory convergence is achieved.

$$
\frac{\partial}{\partial \tau} \mathbf{u}_{N}(\mathbf{x})+\mathcal{D}_{N} \mathbf{u}_{N}(\mathbf{x})+\mathcal{R}\left(\mathbf{u}_{N}(\mathbf{x})\right)=0, \quad \mathbf{x} \in \Omega
$$

Because the collocation scheme uses time-domain data via the discrete Fourier transform, it is possible for the resolved frequency components to be corrupted by aliasing errors [10] in nonlinear equations, e.g. the Navier-Stokes equations governing fluid dynamics. Aliasing errors do not affect the spectral convergence rate of the discrete Fourier series [26], but they can destabilize calculations. Therefore, spectral vanishing viscosity, in the form originally proposed by Tadmor [27], is applied by adding an artificial viscosity term in the form of a second-degree global differentiation operator that is only active above a certain cutoff frequency. This approach damps the high-frequency modes, attenuating their destabilizing impact on the resolved spectrum.

$$
\frac{\partial}{\partial t} u_{N}(\mathbf{x}, t)+\mathcal{R}\left(u_{N}(\mathbf{x}, t)\right)=\epsilon \Delta t \frac{\partial}{\partial t}\left(\mathcal{Q}_{N} \frac{\partial}{\partial t} u_{N}(\mathbf{x}, t)\right), \quad \mathbf{x} \in \Omega
$$

The symmetic viscosity kernel, $\mathcal{Q}_{N}$, is applied only to the higher frequencies by defining a smooth function that is null below the cutoff frequency, $M$, and smoothly transitions to unity at the highest frequencies. Here, the cutoff frequency is taken as roughly two-thirds the highest mode, $M=\left\lfloor\frac{2 K}{3}\right\rfloor$ and coefficient as the reciprocal of the number of harmonics, $\epsilon=K^{-1}$. Huang and Ekici [28] apply spectral vanishing viscosity to dealias HDHB calculations using a binary viscosity kernel. McMullen [29] applies this concept only on the coarse grids within the multigrid framework to more rapidly damp out the high frequency modes without sacrificing accuracy on the finest mesh.

The standard Time-Spectral method outlined above cannot be applied in general within the overset framework. Relative motion of overset grids produces spatial nodes that lack complete time-histories, obviating the ability to uniquely represent the aperiodic solutions with a Fourier series. To circumvent this result, the approach presented by the authors in [16] expands the solution at a given dynamically blanked node within intervals of consecutively unblanked time samples onto a global basis spanning the same sub-periodic interval. Spatial nodes may have multiple associated temporal intervals so solutions are expanded with independent bases within each partition. Here we use barycentric rational interpolants. Fully periodic intervals are still expanded and differentiated in the Fourier basis, resulting in a hybrid approach that employs the optimal basis available. Readers are referred to $[16,30]$ for further detail.

In contrast to the modal complex exponential basis set upon which the spectral vanishing viscosity is based, the nodal basis of the rational interpolant can not be decomposed into modal components to damp only the highest-frequencies. Instead, a dissipation operator derived from barycentric rational interpolants is employed at dynamically-blanked nodes. The dissipation operator is applied only at the two interval boundary nodes to avoid sacrificing accuracy within the interior of the temporal domain.

\section{OVERFLOW}

OVERFLOW is an overset, implicit, finite-difference-based Reynolds-averaged Navier-Stokes (RANS) solver developed at NASA to handle a wide range of flow calculations [31]. To avoid solving the global implicit system at every iteration, OVERFLOW employs an approximate-factorization (AF) of the lefthand-side (LHS) of the discretized equations, whereby each dimension is solved sequentially. The AF scheme [32] requires the inversion of a sequence of either block-tridiagonal or scalar-pentadiagonal matrices, greatly reducing the computational effort required to update the solution at every iteration. The Time-Spectral scheme can be incorporated within this framework by adding an additional factor in the temporal dimension. A brief synopsis of the time-accurate and Time-Spectral discretizations is provided below. 


\section{Temporal Discretization}

OVERFLOW solves the unsteady Navier-Stokes equations in strong conservation-law form in three spatial dimensions.

$$
\frac{\partial Q}{\partial t}+\frac{\partial F}{\partial x}+\frac{\partial G}{\partial y}+\frac{\partial H}{\partial z}=0
$$

The flux vectors, $F, G$ and $H$ are nonlinear functions of the state vector of conserved quantities, $Q$. The equations are presented in Cartesian coordinates, but are solved in generalized curvilinear coordinates.

\section{Time-Accurate Scheme}

In the general case of time-dependent flow, a dual time-stepping scheme is applied to sufficiently reduce the unsteady residual at each physical time step. The time-derivative of the state vector of conserved quantities is approximated with a second-order backward difference formula (BDF2).

$$
\frac{3 Q^{n+1}-4 Q^{n}+Q^{n-1}}{2 \Delta t}+\delta_{x} F^{n+1}+\delta_{y} G^{n+1}+\delta_{z} H^{n+1}=0
$$

The spatial finite-difference operators, $\delta_{x}, \delta_{y}$ and $\delta_{z}$ are applied to the corresponding flux vectors in the $x$-, $y$ - and $z$-directions, respectively. The dual-time advancement scheme is achieved by adding a pseudotime derivative approximated by the first-order backward Euler scheme, with subiteration index $s$.

$$
\frac{Q^{s+1}-Q^{s}}{\Delta \tau}+\frac{3 Q^{s+1}-4 Q^{n}+Q^{n-1}}{2 \Delta t}+\delta_{x} F^{s+1}+\delta_{y} G^{s+1}+\delta_{z} H^{s+1}=0
$$

The flux vectors are nonlinear functions in $Q$ and are therefore linearized about $Q^{s}$. The linearizations are substituted into Eq. 15 which is then put into delta form, where $A, B$ and $C$ are the flux Jacobians in the $x$-, $y$ - and $z$-directions, respectively, $\Delta \tilde{\tau}=\Delta \tau /\left(1+\frac{3 \Delta \tau}{2 \Delta t}\right)$ and $\Delta Q=Q^{s+1}-Q^{s}$.

$$
\left[I+\Delta \tilde{\tau} \delta_{x} A+\Delta \tilde{\tau} \delta_{y} B+\Delta \tilde{\tau} \delta_{z} C\right] \Delta Q=-\Delta \tilde{\tau}\left[\frac{3 Q^{s}-4 Q^{n}+Q^{n-1}}{2 \Delta t}+\delta_{x} F^{s}+\delta_{y} G^{s}+\delta_{z} H^{s}\right]
$$

Approximately factoring the LHS of Eq. 16 results in the final form of the system of equations.

$$
\left[I+\Delta \tilde{\tau} \delta_{x} A\right]\left[I+\Delta \tilde{\tau} \delta_{y} B\right]\left[I+\Delta \tilde{\tau} \delta_{z} C\right] \Delta Q=-\Delta \tilde{\tau}\left[\frac{3 Q^{s}-4 Q^{n}+Q^{n-1}}{2 \Delta t}+\delta_{x} F^{s}+\delta_{y} G^{s}+\delta_{z} H^{s}\right]
$$

The solution is updated by applying a sequence of low-rank inversions onto the right-hand side (RHS) of Eq. 17 on a point-by-point basis. This second-order accurate dual time-stepping procedure is used for the time-accurate calculations presented in Sections IV and V.

\section{Time-Spectral Scheme}

The Time-Spectral discretization is a modification to the discretization of the time-derivative in Eq. 14, where an infinitely-supported spectrally-accurate differentiation operator, $\mathcal{D}_{N}$, replaces an algebraically-accurate finite-difference approximation.

$$
\mathcal{D}_{N} Q^{s+1}+\delta_{x} F^{s+1}+\delta_{y} G^{s+1}+\delta_{z} H^{s+1}=0
$$

The solution vector of conserved variables is now defined over the spatial and temporal dimensions and therefore the spatial and temporal differentiation operators are constructed accordingly, as demonstrated explicitly by Thomas et al. [12] and Naik et al. [33]. As with the time-accurate case, a backward Euler pseudotime advancement scheme is applied to Eq. 18.

$$
\frac{Q^{s+1}-Q^{s}}{\Delta \tau}+\mathcal{D}_{N} Q^{s+1}+\delta_{x} F^{s+1}+\delta_{y} G^{s+1}+\delta_{z} H^{s+1}=0
$$

Linearizations of the flux vectors about $Q^{s}$ are substituted into Eq. 19, which is then put into delta form.

$$
\left[I+\Delta \tau \mathcal{D}_{N}+\Delta \tau \delta_{x} A+\Delta \tau \delta_{y} B+\Delta \tau \delta_{z} C\right] \Delta Q=-\Delta \tau\left[\mathcal{D}_{N} Q^{s}+\delta_{x} F^{s}+\delta_{y} G^{s}+\delta_{z} H^{s}\right]
$$


Finally, the LHS of Eq. 20 is approximately-factored, treating time as an additional direction.

$$
\left[I+\Delta \tau \delta_{x} A\right]\left[I+\Delta \tau \delta_{y} B\right]\left[I+\Delta \tau \delta_{z} C\right]\left[I+\Delta \tau \mathcal{D}_{N}\right] \Delta Q=-\Delta \tau\left[\mathcal{D}_{N} Q^{s}+\delta_{x} F^{s}+\delta_{y} G^{s}+\delta_{z} H^{s}\right]
$$

The solution is updated by a applying a sequence of four directional inversions onto the RHS of Eq. 21. The required modifications to the existing solver are limited to an additional linear solve, of at most dimension $N$, at every grid point for the Time-Spectral AF operator, and an evaluation of the temporal residual term $\left(\mathcal{D}_{N} Q^{s}\right)$ at every grid point. In other words, time is treated in a similar fashion to the spatial independent variables when solving for the steady-state solution in the combined space-time domain.

For spatial nodes with complete time histories, the standard Fourier-based differentiation operator is used for $\mathcal{D}_{N}$. However, for dynamically-blanked nodes, $\mathcal{D}_{N}$ of dimension $N_{P}<N$, is dynamically generated and used for the intervals of $N_{P}$ consecutively-defined time samples. A favorable outcome of the TimeSpectral AF scheme is that the implicit temporal operator, $\left[I+\Delta \tau \mathcal{D}_{N}\right]$, is diagonalized by the discrete Fourier transform if $\Delta \tau$ is constant for each time-sample ( $\Delta \tau$ is still free to change in space enabling local pseudo time stepping). Thus, the temporal update at statically-blanked nodes is reduced from an implicit linear system of equations to a series of scalar equations, removing the need for an inversion of the LHS operator. Replacing the direct linear solution with a fast Fourier transform (FFT) and inverse transform (IFFT) reduces the complexity from $\mathcal{O}\left(N^{3}\right)$ to $\mathcal{O}(N \log N)$ which matches that of the explicit NLFD method $[29]$.

The memory requirements for a Time-Spectral solution are greater than its time-accurate analog because the solution and residual must be stored for each time sample. However, careful implementation limits the storage requirements. Details of memory scaling for the OVERFLOW Time-Spectral solver are outlined in [16] and suggest that a large number of temporal modes can be applied without risk of exhausting the memory budget on suitably parallelized calculations.

\section{Spatial Discretization, Multigrid Acceleration and Turbulence Modeling}

The Time-Spectral approach as applied to OVERFLOW avoids modifying the existing spatial residual and implicit operators because they are applied to each time sample sequentially within each iteration. Secondorder finite differences are used for all of the cases presented, with second- and fourth-difference artificial dissipation using OVERFLOW's default settings of $\epsilon_{2}=2.0$ and $\epsilon_{4}=0.04$ dissipation coefficients. The primary convergence acceleration technique built into OVERFLOW is the Full Approximation Storage (FAS) multigrid algorithm. It is desirable to include multigrid within the Time-Spectral implementation to make simulations of increasingly complex three-dimensional problems more tractable. The FAS spatial multigrid scheme was augmented to include the fully-implicit Time-Spectral scheme on all grid levels. The TimeSpectral multigrid implementation has demonstrated a multifold increase in computational efficiency when applied to representative two-dimensional problems and should greatly assist in the calculation of more complex three-dimensional flows (See Figure 2). No temporal multigrid has been implemented but is included as part of future work.

Approximate-factorization schemes are prone to numerical instabilities in three or more dimensions. The three dimensional Time-Spectral AF scheme applies a sequence of four operators. Thomas et al. [11,12] and Custer [13] demonstrate stability of a two-factor Time-Spectral AF scheme. However, three- and four-factor periodic central-difference AF schemes are unconditionally unstable absent dissipation. Linear stability analysis in [30] demonstrates conditional stability for the Time-Spectral AF scheme in two and three spatial dimensions by applying artificial dissipation to only the spatial operators. This treatment maintains the spectral accuracy of the purely imaginary temporal differentiation operator.

Practical applications of the Time-Spectral method, including rotorcraft and turbomachinery, involve turbulent flows. OVERFLOW employs a loosely coupled turbulence scheme whereby the turbulent variables are updated initially and held constant for the flow-equation iteration. This code structure prevents a direct Time-Spectral implicit treatment for the turbulence variable without a significant overhaul. Instead, a semiimplicit treatment retroactively applies the implicit operator to the semi-implicit turbulent update, $\Delta \tilde{\nu}^{s+\frac{1}{2}}$. Initially, the turbulence equation is updated implicitly in the spatial dimensions where the Time-Spectral temporal residual, $\mathcal{D}_{N} \tilde{\nu}^{s}$ is explicitly added to the RHS.

$$
\left[I+\Delta \tau \delta_{x} A\right]\left[I+\Delta \tau \delta_{y} B\right]\left[I+\Delta \tau \delta_{z} C\right] \Delta \tilde{\nu}^{s+\frac{1}{2}}=-\Delta \tau\left[R\left(\tilde{\nu}^{s}\right)+\mathcal{D}_{N} \tilde{\nu}^{s}\right]
$$

The semi-implicit update, $\tilde{\nu}^{s+\frac{1}{2}}$, is held fixed while advancing the conserved variables from $Q^{s}$ to $Q^{s+1}$. The implicit update, $\Delta \tilde{\nu}^{s+1}$, is computed and retroactively applied to $\tilde{\nu}^{s}$ to advance the solution prior to the 
subsequent iteration.

$$
\left[I+\Delta \tau \mathcal{D}_{N}\right] \Delta \tilde{\nu}^{s+1}=\Delta \tilde{\nu}^{s+\frac{1}{2}}
$$

The one-equation Spalart-Allmaras turbulence model [34] is used for all turbulent RANS cases presented in this work. A limiter is employed on the temporal gradient of the turbulent working variable to prevent a violation of its positivity constraint as demonstrated in [16].

\section{Laminar Plunging Airfoils}

A pair of low speed, low Reynolds number laminar plunging NACA 0012 airfoil cases are investigated as a precursor to the upcoming three-dimensional rotorcraft cases. These flows exhibit some similar physical phenomena to the rotorcraft flows, most notably the convection of strong vortices through the wake. Jones et al. [35] published results from low-speed experiments that serve to highlight the complex nature of the vortical structures associated with these flows as a function of their Strouhal number, $S t=k h$, which is a product of their reduced frequency, $k=\omega c / V_{\infty}$, and nondimensionalized plunging amplitude, $h=a / c$. The instantaneous vertical displacement, $y(t)=h \sin (k t)$, is defined as a single sinusoid of the reduced frequency.

At low Strouhal numbers, the plunging motion results in vortices with a momentum deficit, resulting in a time-averaged drag on the airfoil. As the frequency is increased, the vortical structure changes, eventually resulting in a thrust-producing momentum surplus. Increasing the Strouhal number even further results in both lift and thrust. This behavior has been coined the Knoller-Betz effect after the experimentalists who first independently documented the phenomenon [35]. Rotors in forward flight undergo a host of oscillations, either prescribed or passively via fluid-structure interaction, suggesting that analysis of the two-dimensional problem should uncover computational strategies for the full three-dimensional configurations.

Two primary cases are investigated: a drag-producing case with $S t=0.288$ and a higher-frequency thrustproducing case with $S t=0.6$. Freestream Mach number, $M_{\infty}=0.2$, and Reynolds number, $R e=1850$, are used for both cases. Each case is computed using both rigid- and relative-body motion to compare the performance between the standard and hybrid Time-Spectral methods. The grid system presented in Fig. 3 consists of a $589 \times 33$ C-mesh near-body grid embedded within a hierarchy of isotropic Cartesian off-body grids. The large $909 \times 309$ finest level-one (L1) off-body grid spans $8 c$ downstream with $0.01 c$ grid spacing to accurately resolve a significant portion of the highly-active wake. The hole-cut standoff distance is $0.075 c$ and the Cartesian grids span approximately $200 \mathrm{c}$ to the farfield boundaries in both the $x$ - and $y$-directions.

\section{Drag-Producing Case}

The lower-frequency test case of $S t=0.288$ with reduced frequency $k=3.6$ and plunging amplitude $h=0.08$ results in a vortical wake structure that creates a momentum deficit, producing a time-averaged drag on the airfoil. The case was simulated using time-accurate and Time-Spectral approaches for both rigid and relative motion and qualitatively compared to the experimental results offered in [35]. Figure 4 provides snapshots of the experimental flow field and vorticity magnitude of the simulations. The time-accurate calculation successfully captures the primary flow features depicted in the experimental snapshot and the Time-Spectral simulations for both rigid and relative motion converge to a similar solution, provided the sufficient temporal resolution of around 16 modes. There exist significant variations for the under-resolved Time-Spectral solutions between the rigid- and relative-motion cases, but the underlying wake structure is apparent with just one or two modes for the relative-motion case and just two or four modes for the rigidmotion case. Figure 5 confirms the rapid convergence of the Time-Spectral computed drag coefficient, $c_{d}$, to the time-accurate result. Employing just four modes (See Figure 5b) for either Time-Spectral configuration nearly matches the time-accurate signal. In fact, the relative motion converges slightly faster as evidenced in Fig. 6 where the cyan line corresponding to the rigid-motion case with $N=9$ time-samples lies off the timeaccurate signal for a portion of the period, but the relative-motion signal is identical to the time-accurate signal to the resolution of the figure. Thus, the scalar performance measure of drag converges more rapidly than the vortical flow features.

\section{Thrust-Producing Case}

As the Strouhal number is increased to $S t=0.6$, the vortical wake structure evolves from producing a momentum deficit, as demonstrated for $S t=0.288$, to a momentum surplus, where the time-averaged flow 
exhibits a thrust-inducing jet structure. Selecting a reduced frequency, $k=6.0$ and plunging amplitude $h=0.1$, results in the particular wake structure depicted in Fig. 7. Experimental results taken from [35] and depicted in Fig. 7a use this Strouhal number, $S t=0.6$, except that the reduced frequency is halved and the plunging amplitude doubled $(k=3.0$ and $h=0.2)$. Simulations using these parameters resulted in significantly different vortical wake patterns. Therefore, the case using the higher reduced frequency is employed, which is consistent with the observations and calculations in [36]. Figure 7 demonstrates strong agreement between the time-accurate and experimental data and again demonstrates the convergence of the Time-Spectral solutions for both rigid and relative motion with increased temporal resolution. As with the drag-producing cases, the Time-Spectral calculations require sixteen modes to qualitatively match the time-accurate solution. The bulk features of the wake are resolved using half as many modes, but the solutions do not match in the vicinity of the trailing edge. Figure 8 confirms the convergence of the TimeSpectral computed drag coefficient, $c_{d}$, to the time-accurate result for the thrust-producing case. Seventeen time-samples are sufficient to reproduce the time-accurate result for both the rigid- and relative-motion Time-Spectral calculations (See Figure 9).

Unlike the drag-producing case using $k=0.288$, spectral vanishing viscosity is required to adequately converge the Time-Spectral simulations for the thrust-producing configuration for both the rigid- and relativebody motion calculations. Initially, spectral vanishing viscosity is only applied to the statically-blanked (Fourier-based) nodes in the case of relative motion, leaving the dynamically-blanked nodes free of any added dissipation. For the relative motion case with $N=17$, additional dissipation is required at the undamped, dynamically-blanked nodes as described in $\S I I$.

Comparison of the rigid- and relative-motion Time-Spectral simulations for the low-speed laminar NACA 0012 suggest similar convergence to the time-accurate result as a function of the number of temporal degrees of freedom. However, the plunging amplitudes for these cases are relatively small. The inviscid plunging NACA 0012 airfoil case presented in [16], with a significantly larger plunging amplitude of $h=0.5$, demonstrates that significantly more temporal modes are required to adequately resolve the flow for the relative-motion Time-Spectral simulation. The sub-optimal basis used to describe the solution at dynamically-blanked nodes is likely partially responsible for this result, but so too is the fact that the stationary background grid is not moving with the body. The solution on the background grid remains periodic, however, its frequency content is driven by the relative motion between itself and the plunging near-body grid. Consider the solution at a point on the near-body grid moving with the airfoil. Over the course of the period of motion, it experiences smooth transitions between compressions and expansions. While a stationary point on the background grid in the vicinity of the airfoil experiences periodic compressions and expansions, the transitions become more rapid as the plunging amplitude increases; the airfoil moves past the stationary point with a larger velocity and therefore the transition occurs over a shorter duration. This increases the frequency of the response that must be approximated by the discrete Fourier series. Therefore, more modes may need to be retained in order to resolve the relative-motion case because its solution contains higher-harmonics on the stationary grid.

Figures 10 and 11 provides the time and frequency response (of streamwise momentum, $\rho u$ ) of the subsonic inviscid plunging airfoil at a node located approximately $0.35 c$ downstream of the trailing edge on both the near- and off-body grids in the neutral position. For the case of rigid-body motion, the off-body grid translates with near-body grid and the nodes remain approximately coincident for all time. For the case of relative motion, the node on the off-body grid remains in place and is therefore only approximately coincident with the node on the near-body grid at two time instances over the period of oscillation. The higher-frequency content in the solution at a node on the stationary background grid suggests that more frequencies are required to resolve the solution using relative motion than with rigid motion. This assertion is supported by Fig. 12 that plots the reconstruction of the off-body grid solution from the frequency components defined by a discrete Fourier transform of the time-accurate solution. Figure 12a demonstrates that roughly four modes are sufficient to adequately resolve the solution at the off-body grid point for the case of rigid-body motion. However, the reconstructions plotted in Fig. 12b suggest that thirty or more modes are required to sufficiently resolve the flow at the stationary off-body grid point. However, many fewer modes are required to match the time-accurate result with respect to integrated loads such as drag [16], supporting the TimeSpectral method as a potential reduced-order model (ROM). For such simple cases, rigid motion would naturally be a more efficient choice as fewer modes are required to resolve the flow. However, more complex configurations (e.g. multiple independently oscillating airfoils) would require a relative motion treatment for a non-deforming grid. The transmission of low-frequency signals in one frame to high-frequency signals in 
another frame proves fundamental to the case of rotorcraft, which is explored in the upcoming section.

\section{Three-Dimensional V-22 Osprey Tiltotor}

The present section demonstrates the ability of the proposed Time-Spectral approach to successfully match time-accurate predictions of a realistic three-dimensional application - the isolated V-22 Osprey tiltrotor depicted in Fig. 13a. Rotorcraft performance is primarily assessed on the converged periodic steadystate flowfields of both hover and forward flight, and therefore both cases are included. ${ }^{a}$ Selection of the highstiffness and relatively low aspect ratio V-22 rotor permits the assumption of non-deforming blades, removing the need to incorporate aeroelastic effects. However, aeroelasticity is a critical component of rotorcraft performance analysis in general and should be included either directly, in a fluid-structure formulation, or with a comprehensive coupling procedure. Research on aeroelasticity in the context of the Time-Spectral method is ongoing $[9,21,37,38]$, but including aeroelasticity is beyond the current scope. Additionally, the V-22 rotor is not typically used in forward (edgewise) flight as it operates in either hover mode or airplane (propeller) mode. Nevertheless, forward-flight Time-Spectral results are compared to those computed in an unsteady environment to evaluate the predictive capabilities of the Time-Spectral approach for that flight regime.

\section{Hover}

The V-22 Osprey tiltrotor has been analyzed for hover extensively - both experimentally and numerically. The quarter-scale Tilt Rotor Aeroacoustic Model (TRAM) was tested at the Duits-Nederlandse Windtunnel (DNW) with experimental details provided in $[39,40]$. The isolated rotor geometry is used for all calculations presented which is consistent with the numerical experiments detailed in $[41,42,43,44]$. With respect to overset grids, the typical solution procedure involves revolving the body-fitted curvilinear grids through a system of stationary background grids. Despite the relatively low aspect ratio of the V-22 blades, a successful Time-Spectral simulation using this approach would require retaining a large number of harmonics due to the high-frequency response induced on the fixed background grids by the relatively low-frequency signals on the near-body grids moving with the blades. This is analogous to the two-dimensional plunging airfoil result shown in Fig. 11 where a relatively low-frequency signal on moving near-body grids induces a much higher-frequency response on stationary background grids. Alternatively, rigid-body motion can be achieved by rotating the background grids with the rotor blades. Since hover can be well estimated as a steady flow in the rotating frame (See [41] for non-inertial reference frame results), it is expected that only a limited number of temporal modes are required when all grids rotate in unison. However, employing rigid-body motion fails to introduce any dynamically-blanked nodes. The case of hover is used to validate the threedimensional OVERFLOW Time-Spectral implementation. Dynamically-blanked nodes are introduced in the forward-flight case discussed in the upcoming section.

The objective of the hover test case is to validate the three-dimensional Time-Spectral OVERFLOW solver against the standard OVERFLOW time-accurate solver, by using the same grid systems and spatial discretizations to isolate the effects of varying the temporal discretization. The grid system includes three blades, each composed of an C-mesh blade grid and two cap-grids, one each for the root and tip, as depicted in Fig. 14a. The C-mesh consists of $181 \times 94 \times 65$ points in the chordwise, radial and body-normal directions, respectively. Each blade, root and tip grid contains 1.1, 0.48 and 0.33 million grid points, respectively, for a total of 5.7 million near-body grid points. The off-body grid system, depicted in Fig. 14b, is comprised of a hierarchy of forty-six isotropic Cartesian grids spanning 15 rotor radii $(\mathrm{R})$ to the farfield boundaries in each direction, totaling 21.3 million mesh points. Thus the total number of grid points is 27.1 million. The finest Cartesian L1 grid spans roughly $\pm 1.2 \mathrm{R}$ in both the $x$ - and $y$-directions and $-1.9 \mathrm{R}$ and $+0.38 \mathrm{R}$ in the $z$-direction, with spacing of $0.1 c_{\text {tip }}$. While a center body is included in the experiment and previous computations, it is omitted in the current work.

Previous numerical calculations of the V-22 TRAM hover case employed a series of turbulence model treatments, but the current results were computed following the laminar off-body procedure outlined by Potsdam and Pulliam [42]. The one-equation SA turbulence model is solved on all body-conforming near-

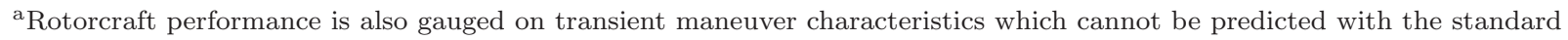
Time-Spectral method. Mavriplis et al. [24] extended the Time-Spectral method to treat quasi-periodic flows like rotorcraft maneuver by applying periodic subtraction. 
body grids. However, the turbulence model production source terms are deactivated on the Cartesian background grids to prevent the eddy viscosity from growing unbounded in the vortex-dominated wake and feeding back to the flow in the vicinity of the rotor.

The hover test case uses a uniform collective of $14^{\circ}$ with a tip speed of $M_{\text {tip }}=0.625$ and tip Reynolds number of $R e_{\text {tip }}=2.1$ million, which is consistent with the primary TRAM test case in [42]. Qualitative results from both time-accurate and single-harmonic Time-Spectral V-22 TRAM hover calculations are presented in Figs. 15-16. Figure 15 plots iso-contours of vorticity magnitude, as well as a cutting plane of the same functional. Figure 16 plots the same iso-contours of vorticity magnitude over a cutting plane of undampled eddy viscosity. The time-accurate and Time-Spectral calculations demonstrate strong agreement in both cases.

In general, dozens of periods are required to converge certain functionals of interest in a time-accurate calculation. The Figure of Merit, $F M$, is an established scalar performance measure for rotorcraft in hover that measures rotor efficiency; it is a ratio of the ideal power, derived from momentum theory, to the actual (computed) power.

$$
F M=\frac{1}{\sqrt{2}} \frac{C_{T}^{3 / 2}}{C_{Q}}
$$

The Time-Spectral simulation is steady in nature and converges directly to the space-time solution.

\begin{tabular}{cccc} 
& $C_{T}$ & $C_{Q}$ & $F M$ \\
\hline TS & $0.11379 \times 10^{-1}$ & $0.15320 \times 10^{-2}$ & 0.5602 \\
TA & $0.11383 \times 10^{-1}$ & $0.15320 \times 10^{-2}$ & 0.5605 \\
\hline
\end{tabular}

Table 1: Isolated V-22 Osprey Tiltrotor in Hover. Force and moment coefficients and Figure of Merit for the single harmonic Time-Spectral (TS) and time-accurate (TA) calculations.

Table 1 provides the force and moment coefficients and the Figure of Merit for the time-accurate and single harmonic Time-Spectral calculations. The Figure of Merit computed using the Time-Spectral method with a single harmonic agrees with the time-accurate result to five percent of a percent. However, the Figure of Merit is substantially lower than those presented in $[41,42,43,44]$. This discrepancy is attributable the use of only second-order central differencing of the convective terms, as opposed to higher-order schemes in the references cited. Preliminary results do indicate an improvement with increasing the spatial discretization order of accuracy. Nevertheless, the case of hover has validated the three-dimensional Time-spectral implementation.

\section{Forward Flight}

The hover case of the previous section prescribed the rigid motion of the isolated V-22 rotor with its offbody grid system, resulting in an absence of dynamically-blanked nodes. Rotors in forward (edgewise) flight experience an imbalance of dynamic pressure on the advancing $(0 \leq \Psi \leq 180)$ and retreating $(180 \leq \Psi \leq 360)$ segments of a rotation (See Fig. 13b). Left untreated, this leads to an undesired rolling moment about the longitudinal axis of the vehicle. Cyclic pitch is used to mitigate the force imbalance by dynamically changing the pitching amplitude of the blade as it rotates. On the advancing side, where blades experience a higher dynamic pressure, pitch is decreased to reduce the resulting normal force. Conversely, blades pitch upwards on the retreating side to increase the normal force in the presence of lower dynamic pressure. While the background grids are still prescribed to rotate with the rotor, the relative pitching motion between the blades and background grids results in dynamically-blanked nodes, thereby requiring the hybrid Time-Spectral scheme.

While the V-22 Osprey is not typically employed in forward (edgewise) flight because its thrust is derived by tilting its rotors forward into propellor mode, the edgewise-flight case provides an opportunity to gauge the three-dimensional hybrid Time-Spectral scheme for a rigid blade. A reasonable selection of parameters are selected for collective and cyclic pitch values estimated from [39]. Additionally, the rotor shaft axis is chosen to be vertical. While the rotor is not trimmed (e.g. with a comprehensive analysis tool), the prescription of fixed collective and cyclic pitch serve as a representative estimate of rotor trim. Additionally, the rotors move in prescribed fashion, ignoring the force-induced blade dynamics typically associated with high-fidelity rotorcraft calculations. The current objective is to compare Time-Spectral results to those computed with 
the standard time-accurate flow solver. Aeroelasticity and deformation are complications that obscure the current objective, but should be included in full-scale rotorcraft simulations.

Time-accurate and Time-Spectral forward-flight V-22 TRAM calculations are presented for an advance ratio, $\mu=0.2$, where $M_{\infty}=\mu M_{\text {tip }}$, using the same tip Mach and Reynolds numbers as the hover case. The test case employs a fixed collective, $\theta_{0}=10^{\circ}$, and longitudinal and lateral cyclic pitch amplitudes, $\theta_{C 1}=3^{\circ}$ and $\theta_{S 1}=-5^{\circ}$, respectively, where, the pitch amplitude, $\theta(\Psi)$, is prescribed by the following relationship.

$$
\theta(\Psi)=\theta_{0}+\theta_{C 1} \cos (\Psi)+\theta_{S 1} \sin (\Psi)
$$

Azimuthal angle, $\Psi$, is taken to be zero in the free stream direction as depicted in Fig. 13b. The off-body grid system is modified for the forward flight case because vorticity shed off of the rotor is convected downstream as opposed to straight down as for the case of hover. Thus, the Cartesian off-body grid system is compressed to provide a wider L1 grid near the rotor to capture vortical content as it convects downstream. The off-body grid system is still comprised of a hierarchy of 46 isotropic Cartesian meshes combining for a total of 20.3 millions grid points. Therefore, the entire grid system is comprised of 26.1 million grid points, which is nearly equivalent to the amount used for hover. The L1 grid spans roughly $\pm 1.65 \mathrm{R}$ in both the $x$ - and $y$-directions and $-0.9 \mathrm{R}$ and $+0.28 \mathrm{R}$ in the $z$-direction, with the same spacing of $0.1 c_{\text {tip }}$ as the hover case. Additionally, spectral vanishing viscosity is required to satisfactorily converge the Time-Spectral calculations.

Qualitative comparisons between the time-accurate and Time-Spectral forward flight results are evident in Figs. 17-18. Figure 17 plots iso-contours of vorticity magnitude and a cutting plane of undamped eddy viscosity. The Time-Spectral solution using only $N=11$ time samples shows some agreement with the time-accurate result but demonstrates marked disagreement in the turbulence variable. The Time-Spectral solution using $N=21$ time samples offers significant improvement. The $N=31$ Time-Spectral solution resolves of some of the finer features exhibited in the time-accurate result and shows improved agreement of the turbulent eddy viscosity field. The side views of the four cases, provided in Fig. 18, demonstrate similar trends. Unlike the case of hover, the flow is no longer steady in the rotating frame. The tip vortices being convected downstream pose a resolution problem for unsteady simulation as a point on the rotating background mesh moves quickly through the vortices. This is yet another example of a case where a relatively low-frequency disturbance in one frame is transformed to a much higher frequency signal in another frame, which may explain the poor resolution of the tip vortices in the Time-Spectral calculations.

Finally, Figure 19 demonstrates quantitative convergence of the Time-Spectral calculations to the timeaccurate solution for the instantaneous thrust coefficient, $C_{T}$, versus azimuthal angle, $\Psi$. Despite rather poor qualitative agreement, the $N=11$ Time-Spectral case resolves the bulk $C_{T}$ signal. Naturally, agreement improves as the temporal resolution is refined. The $N=31$ Time-Spectral result matches the time-accurate signal over nearly the entire period.

\section{Summary \& Future Work}

A brief synopsis of the Time-Spectral was provided with implementation details within NASA's implicit OVERFOW RANS solver. The Time-Spectral OVERFLOW solver was applied to problems in two and three dimensions. Investigation of low-speed laminar plunging airfoils demonstrated the ability of the standard and hybrid Time-Spectral schemes to accurately simulate solutions with highly-vortical wakes over a range of reduced frequencies. The Time-Spectral solver was then used to demonstrate successful calculations of three dimensional rotorcraft problems for the isolated V-22 Osprey rotor in hover and forward (edgewise) flight. Only a single harmonic is required to resolve the hover cases where all grids rotate in unison. The more active wake associated with forward flight requires significantly more temporal resolution.

With the goal of improving efficiency, future work includes parallelization in the temporal dimension [24] and a zone-based frequency adaptation strategy to more optimally allocate the temporal degrees of freedom to more dynamic regions of the flow. Frequency adaptation in the context of temporal Fourier schemes has been developed $[45,46,47,48,49,50,51]$, however refinement on a point-by-point basis is not practical in the context of overset grids. Rather, the adaptive mesh refinement model can be employed in time so that every grid has an associated number of time-instances that can be refined as the simulation advances. New grids can also be generated to resolve high-frequency regions of the flow. Spatio-temporal multigrid also offers potential convergence acceleration and will be included as part of future work. 


\section{Acknowledgments}

The authors would like to thank the US Army Aeroflightdynamics Directorate (AMRDEC) for sponsoring this research and the NASA Education Associates Program (EAP).

\section{References}

${ }^{1}$ Hall, K., Thomas, J., and Clark, W., "Computation of Unsteady Nonlinear Flows in Cascades using a Harmonic Balance Technique," 9th International Symposium on Unsteady Aerodynamics, Aeroacoustics and Aeroelasticity of Turbomachines, Lyon, France, September 2000.

${ }^{2}$ Hall, K., Thomas, J., and Clark, W., "Computation of Unsteady Nonlinear Flows in Cascades Using a Harmonic Balance Technique," AIAA Journal, Vol. 40, May 2002, pp. 879-886.

${ }^{3}$ McMullen, M. S. and Jameson, A., "The Computational Efficiency of Non-Linear Frequency Domain Methods," Journal of Computational Physics, Vol. 212, 2006, pp. 637-661.

${ }^{4}$ McMullen, M., Jameson, A., and Alonso, J., "Demonstration of Nonlinear Frequency Domain Methods," AIAA Journal, Vol. 44, No. 7, July 2006, pp. 1428-1435.

${ }^{5}$ Nadarajah, S. K., McMullen, M. S., and Jameson, A., "Optimum Shape Design for Unsteady Flow Using Time Accurate and Non-Linear Frequency Domain Methods," AIAA Paper 3875, June 2003.

${ }^{6}$ Murman, S. M., "A Reduced-Frequency Approach for Calculating Dynamic Derivatives," AIAA Journal, Vol. 45, No. 6, June 2007, pp. 1161-1168.

${ }^{7}$ Gopinath, A. K. and Jameson, A., "Time Spectral Method for Periodic Unsteady Computations over Two- and ThreeDimensional Bodies," AIAA Paper 1220, January 2005.

${ }^{8}$ Gopinath, A. K. and Jameson, A., "Application of the Time Spectral Method to Periodic Unsteady Vortex Shedding," AIAA Paper 0449, January 2006.

${ }^{9}$ Blanc, F., Roux, F.-X., Jouhaud, J.-C., and Boussuge, J.-F., "Numerical Methods for Control Surfaces Aerodynamics with Flexibility Effects," IFASD 2009, Seattle, WA, June 2009.

${ }^{10}$ Canuto, C., Hussaini, M. Y., Quarteroni, A., and Zang, T. A., Spectral Methods - Fundamentals in Single Domains, Scientific Computation, Springer, Germany, 2006.

${ }^{11}$ Thomas, J. P., Custer, C. H., Dowell, E. H., and Hall, K. C., "Unsteady Flow Computation Using a Harmonic Balance Approach Implemented about the OVERFLOW 2 Flow Solver," AIAA Paper 4270, June 2009.

${ }^{12}$ Thomas, J. P., Custer, C. H., Dowell, E. H., and Hall, K. C., "Compact Implementation Strategy for a Harmonic Balance Method Within Implicit Flow Solvers," AIAA Journal, Vol. 51, No. 6, June 2013, pp. 1374-1381.

${ }^{13}$ Custer, C. H., A Nonlinear Harmonic Balance Solver for an Implicit CFD Code: OVERFLOW 2, Ph.D. thesis, Duke University, 2009.

${ }^{14}$ Soucy, O. and Nadarajah, S. K., "A NLFD Method for the Simulation of Periodic Unsteady Flows for Overset Meshes," AIAA Paper 4271, McGill University, June 2009.

${ }^{15}$ Mavriplis, D., Yang, Z., and Mundis, N., "Extensions of Time Spectral Methods for Practical Rotorcraft Problems," AIAA Paper 0423, January 2012.

${ }^{16}$ Leffell, J. I., Murman, S. M., and Pulliam, T. H., "An Extension of the Time-Spectral Method to Overset Solvers," AIAA Paper 0637, Grapevine, Texas, January 2013.

${ }^{17}$ Tatossian, C. A., Nadarajah, S. K., and Castonguay, P., "Aerodynamic Shape Optimization of Hovering Rotor Blades Using a Non-Linear Frequency Domain Approach," AIAA Paper 0322, McGill University, Reno, Nevada, January 2008.

${ }^{18}$ Nadarajah, S. K. and Tatossian, C. A., "Adjoint-Based Aerodynamic Shape Optimization of Rotorcraft Blades," AIAA Paper 6730, August 2008.

${ }^{19}$ Ekici, K., Hall, K. C., and Dowell, E. H., "Computationally Fast Harmonic Balance Methods for Unsteady Aerodynamic Predictions of Helicopter Rotors," AIAA Paper 1439, Duke University, Reno, Nevada, January 2008.

${ }^{20}$ Choi, S., Potsdam, M., Lee, K., Iaccarino, G., and Alonso, J. J., "Helicopter Rotor Design Using a Time-Spectral and Adjoint-Based Method," AIAA Paper 5810, September 2008.

${ }^{21}$ Choi, S. and Datta, A., "CFD Prediction of Rotor Loads using Time-Spectral Method and Exact Fluid-Structure Interfaceal Method and Exact Fluid-Structure Interface," AIAA Paper 7325, Honolulu, Hawaii, August 2008.

${ }^{22}$ Yang, Z., Mavriplis, D. J., and Sitaraman, J., "Prediction of Helicopter Maneuver Loads Using BDF/Time Spectral Method on Unstructured Meshes," AIAA Paper 1122, University of Wyoming, Orlando, Florida, January 2011.

${ }^{23}$ Caradonna, F. and Tung, C., Experimental and Analytical Studies of a Model Helicopter Rotor in Hover, Technical Manual NASA TM-81232, NASA Ames Research Center, Moffett Field, CA, September 1981.

${ }^{24}$ Mavriplis, D. J. and Yang, Z., "Time Spectral Method for Periodic and Quasi-Periodic Unsteady Computations on Unstructured Meshes," AIAA Paper 5034, University of Wyoming, Chicago, Illinois, June 2010.

${ }^{25}$ Peyret, R., Spectral Methods for Incompressible Viscous Flow, Vol. 148 of Applied Mathematical Sciences, SpringerVerlang, New York, 2002.

${ }^{26}$ Snider, A. D., "An Improved Estimate of the Accuracy of Trigonometric Interpolation," SIAM Journal of Numerical Analysis, Vol. 9, No. 3, September 1972, pp. 505-508.

${ }^{27}$ Tadmor, E., "Convergence of Spectral Methods for Nonlinear Conservation Laws," SIAM Journal of Numerical Analysis, Vol. 26, No. 1, February 1989, pp. 30-44.

${ }^{28}$ Huang, H. and Ekici, K., "Stabilization of High-Dimensional Harmonic Balance Solvers Using a Temporal Spectral Viscosity Operator," AIAA Paper 0354, University of Tennessee, Grapevine, Texas, January 2013. 
${ }^{29}$ McMullen, M. S., The Application of Non-Linear Frequency Domain Methods to the Euler and Navier-Stokes Equations, Ph.D. thesis, Stanford University, Stanford, CA, March 2003.

${ }^{30}$ Leffell, J. I., An Overset Time-Spectral Method for Relative Motion, Ph.D. thesis, Stanford University, June 2014.

${ }^{31}$ Nichols, R., Tramel, R., and Buning, P., "Solver and Turbulence Model Upgrades to OVERFLOW 2 for Unsteady and High-Speed Applications," AIAA Paper 2824, 2006.

${ }^{32}$ Beam, R. M. and Warming, R., "An Implicit Finite-Difference Algorithm for Hyperbolic Systems in Conservation-Law Form," Journal of Computational Physics, Vol. 22, 1976, pp. 87-110.

${ }^{33}$ Naik, K. R., Economon, T. D., Colonno, M. R., Palacios, F., and Alonso, J. J., "A Time-Spectral Adjoint Formulation for Shape Optimization of Helicopter Rotors in Forward Flight," AHS Paper 082, Stanford University, Phoenix, Arizona, May 2013.

${ }^{34}$ Spalart, P. and Allmaras, S., "A One-Equation Turbulence Model for Aerodynamic Flows," AIAA Paper 0439, Boeing Commercial Airplane Group, Reno, NV, January 1992.

${ }^{35}$ Jones, K. D., Dohring, C. M., and Platzer, M. F., "Experimental and Computational Investigation of the Knoller-Betz Effect," AIAA Journal, Vol. 36, No. 7, July 1998, pp. 1240-1246.

${ }^{36}$ Allaneau, Y. and Jameson, A., "Direct Numerical Simulations of Plunging Airfoils," AIAA Paper 728, Stanford University, Orlando, Florida, January 2010.

${ }^{37}$ Mundis, N. L. and Mavriplis, D. J., "Quasi-periodic Time Spectral Method for Aeroelastic Flutter Analysis," AIAA Paper 0638, University of Wyoming, Grapevine, Texas, January 2013.

${ }^{38}$ Mundis, N. L. and Mavriplis, D. J., "An Efficient Flexible GMRES Solver for the Fully-coupled Time-Spectral Aeroelastic System," AIAA Paper 1427, University of Wyoming, National Harbor, Maryland, January 2014.

${ }^{39}$ Young, L. A., Earl R. Booth, J., Yamauchi, G. K., Botha, G., and Dawson, S., "Overview of the Testing of a Small-Scale Proprotor," American Helicopter Society $55^{\text {th }}$ Annual Forum, Montreal, Canada, May 1999.

${ }^{40}$ Swanson, S. M., McCluer, M. S., Yamauchi, G. K., and Swanson, A. A., "Airloads Measurements From a 1/4-Scale Tiltrotor Wind Tunnel Test," $25^{\text {th }}$ European Rotorcraft Forum, Rome, Italy, September 1999.

${ }^{41}$ Potsdam, M. A. and Strawn, R. C., "CFD Simulations of Tiltrotor Configurations in Hover," Journal of the American Helicopter Society, Vol. 50, No. 1, 2005, pp. 82-94, Also presented at the American Helicopter Society $58^{t h}$ Annual Forum, Montreal, Canada, June 11-13, 2002.

${ }^{42}$ Potsdam, M. and Pulliam, T., "Turbulence Modeling Treatment for Rotorcraft Wakes," Presented at the AHS Specialist's Conference on Aeromechanics, San Francisco, CA, January 2008.

${ }^{43}$ Chaderjian, N. M. and Buning, P. G., "High Resolution Navier-Stokes Simulations of Rotor Wakes," Proceedings of the American Helicopter Society $67^{\text {th }}$ Annual Forum, Virginia Beach, VA, May 2011.

${ }^{44}$ Chaderjian, N. M., "Advances in Rotor Performance and Turbulent Wake Simulation using DES and Adaptive Mesh Refinement," Seventh International Conference of Computational Fluid Dynamics ICCFD7-3506, NASA Ames Research Center, Big Island, Hawaii, July 2012.

${ }^{45}$ Maple, R. C., Adaptive Harmonic Balance Method for Unsteady, Nonlinear, One-Dimensional Periodic Flows, Ph.D. thesis, The Air Force Institute of Technology, Wright-Patterson Air Force Base, Ohio, September 2002.

${ }^{46}$ Maple, R. C., King, P. I., Wolff, J. M., and Orkwis, P. D., "Split-Domain Harmonic Balance Solutions to Burger's Equation for Large-Amplitude Distrubances," AIAA Journal, Vol. 41, No. 2, February 2003, pp. 206-212.

${ }^{47}$ Maple, R. C., King, P. I., and Oxley, M. E., "Adaptive Harmonic Balance Solutions to Euler's Equation," AIAA Journal, Vol. 41, No. 9, September 2003, pp. 1705-1714.

${ }^{48}$ Mosahebi, A. and Nadarajah, S. K., "An Adaptive Nonlinear Frequency Domain Method for Viscous Periodic State Flows," AIAA Paper 1267, McGill University, Orlando, Florida, January 2010.

${ }^{49}$ Mosahebi, A. and Nadarajah, S. K., "An Implicit Adaptive Non-Linear Frequency Domain Method ( $p$ NLFD) for Viscous Periodic Steady State Flows on Deformable Grids," AIAA Paper 0775, January 2011.

${ }^{50}$ Mosahebi, A. and Nadarajah, S. K., "Algorithmic Advances for the Adapive Non-Linear Frequency Domain Method," AIAA Paper 380, McGill University, Grapevine, Texas, January 2013.

${ }^{51}$ Mosahebi, A. and Nadarajah, S. K., "An Adaptive Non-Linear Frequency Domain Method for Viscous Flows," Computers E Fluids, Vol. 75, April 2013, pp. 140-154.

${ }^{52}$ Leishman, J., Principle of Helicopter Aerodynamics, Cambridge University Press, 2000.

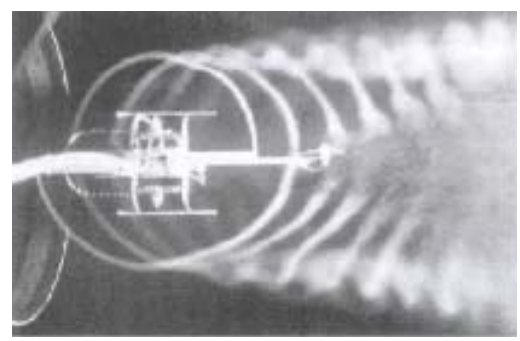

(a) Experimental

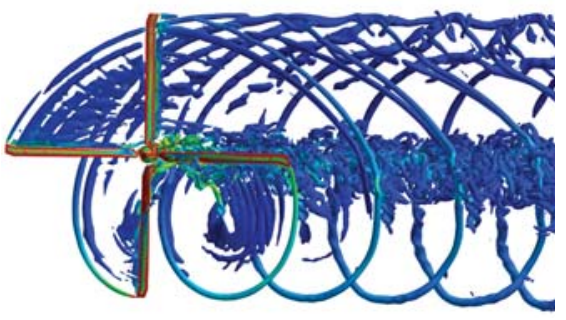

(b) Computational

Figure 1: Top view of rotorcraft forward flight flow characteristics (a) experimental from [52] and (b) numerical simulation of a high-speed UH-60 case C8534, with $M_{\infty}=0.236$ and $\mu=0.37$ using 61 million grid points [44]. 


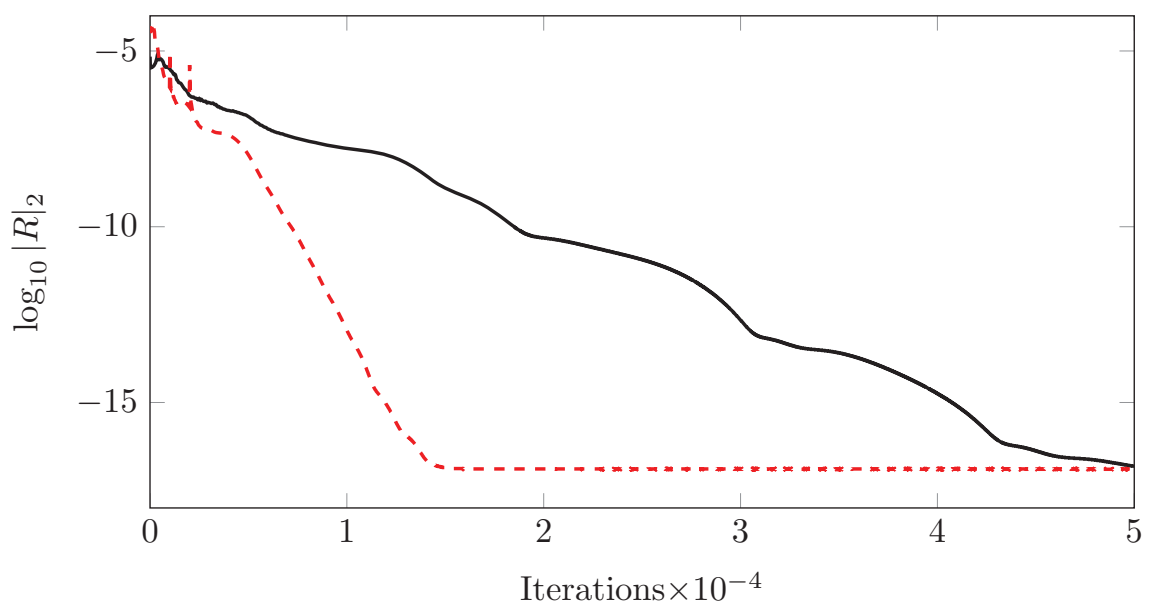

(a) Space-time residual

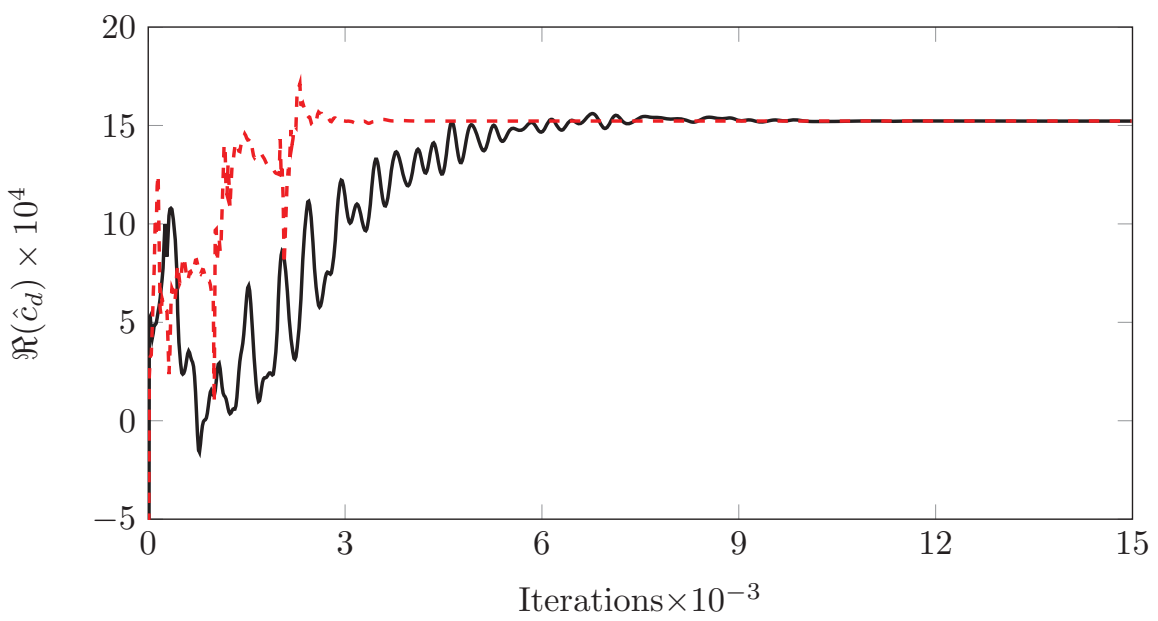

(b) Real component of $c_{d}$ for first harmonic

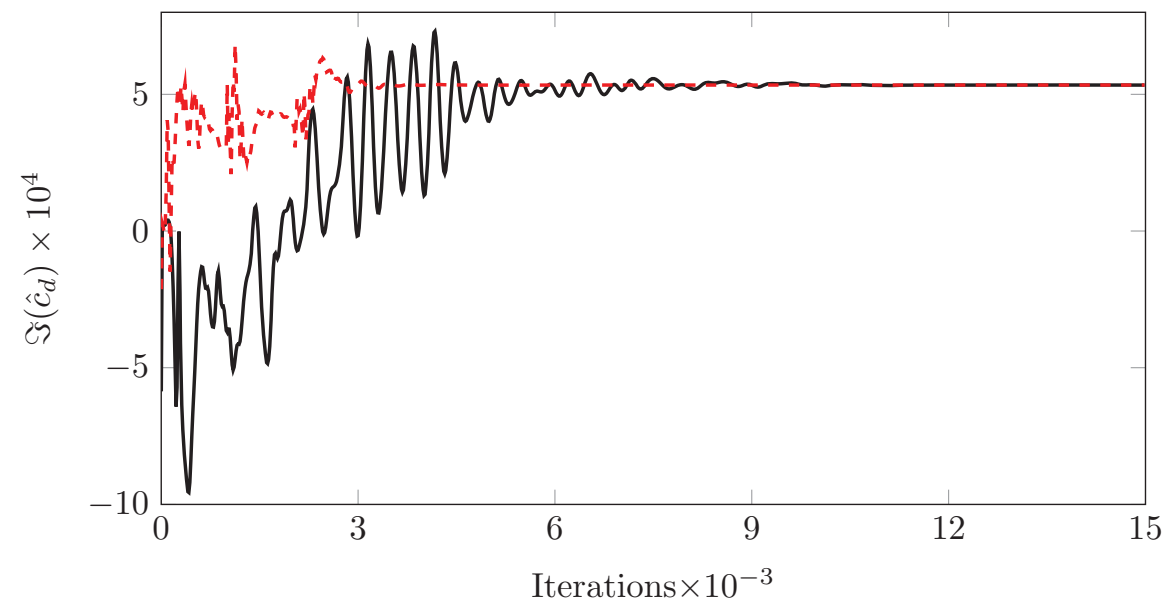

(c) Imaginary component of $c_{d}$ for first harmonic

Figure 2: Residual and drag coefficient convergence for a single grid (black line) versus three-level full multigrid (hashed red line) for a laminar plunging airfoil case with $S t=0.288$. 


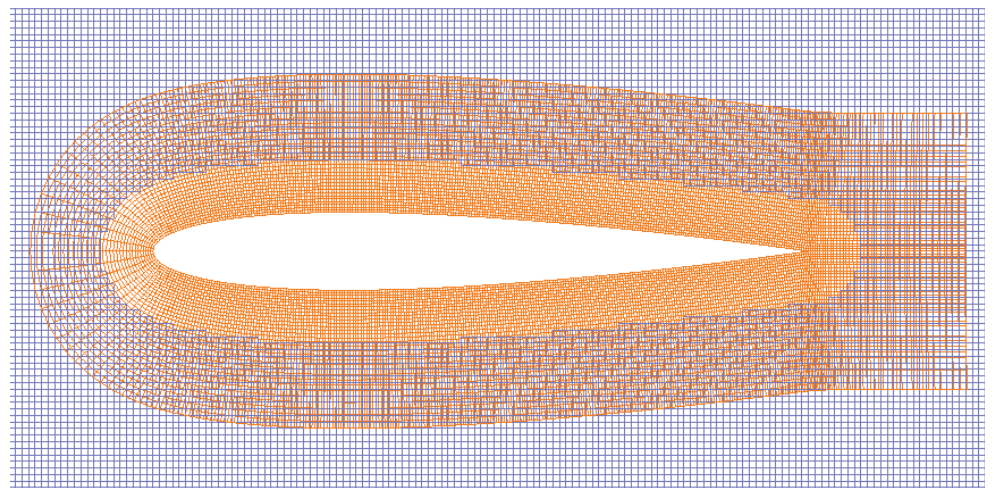

(a) Near-body grid C-mesh with hole-cut.

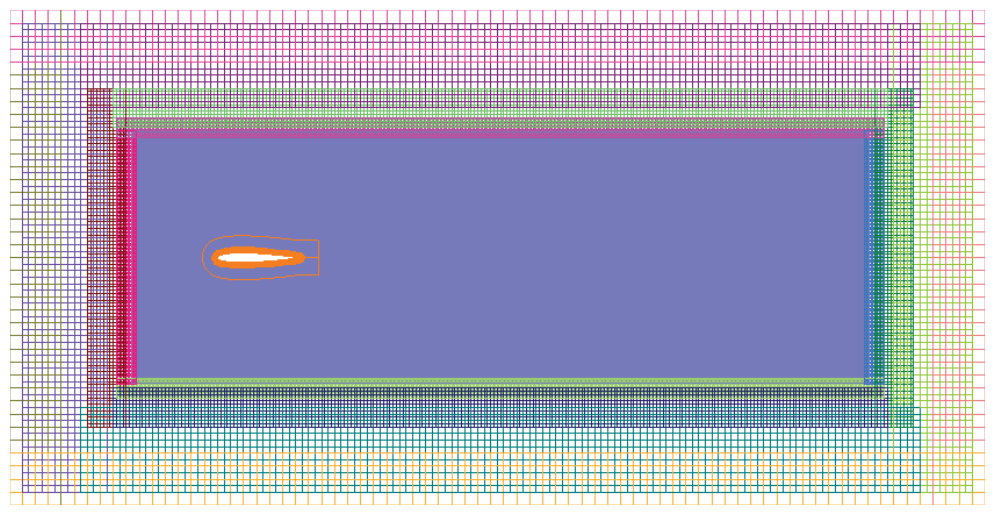

(b) Cartesian off-body grid structure.

Figure 3: Laminar Plunging NACA 0012 Airfoil. Grid system. 


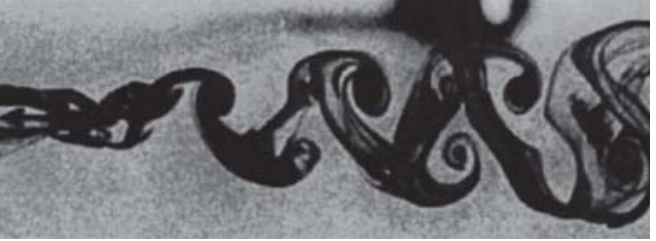

(a) Experimental [35]

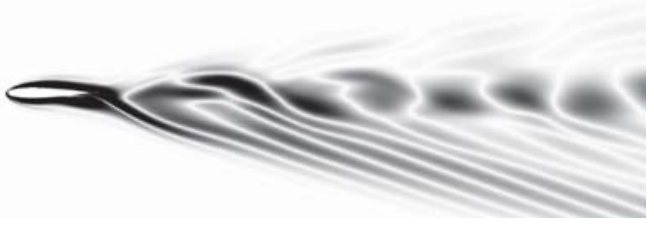

(c) Rigid, $N=3$

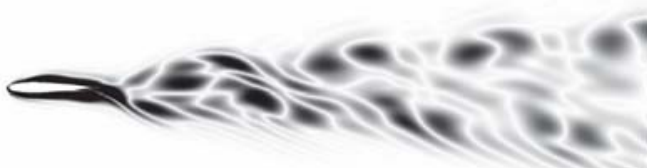

(e) Rigid, $N=5$

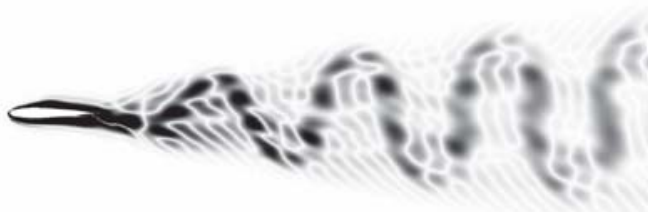

(g) Rigid, $N=9$

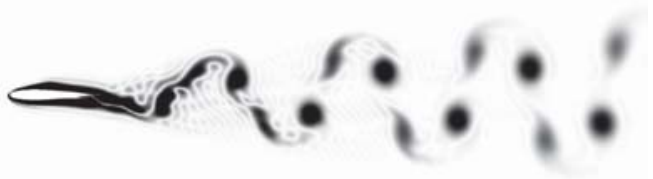

(i) Rigid, $N=17$

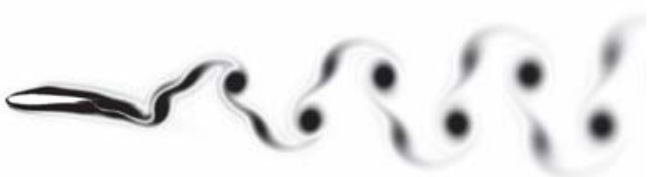

(k) Rigid, $N=33$

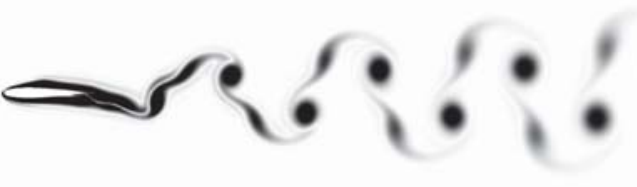

(b) Time Accurate

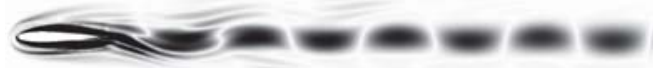

(d) Relative, $N=3$

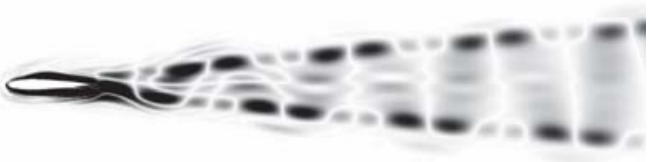

(f) Relative, $N=5$

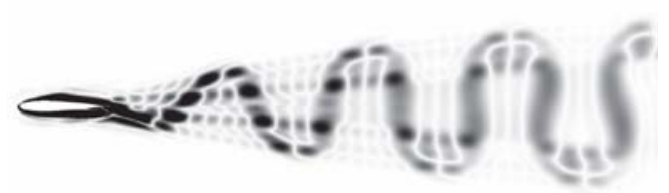

(h) Relative, $N=9$

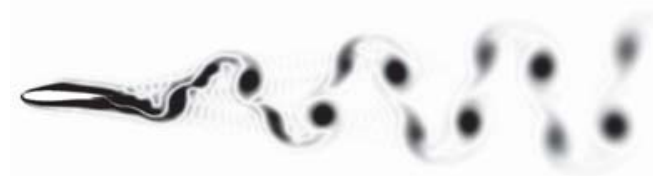

(j) Relative, $N=17$

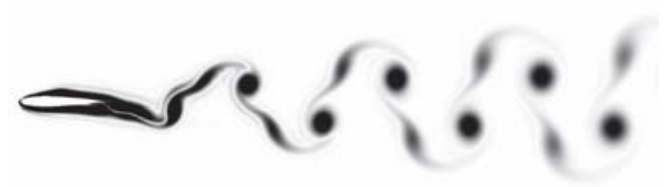

(l) Relative, $N=33$

Figure 4: Laminar Plunging NACA 0012 Airfoil. Drag-producing case at $M_{\infty}=0.2$ and $R e=1850$. Visualization of vorticity magnitude. Comparison of experimental with computed time-accurate and Time-Spectral results using $N \in\{3,5,9,17,33\}$ for both rigid- and relative-body motion. Note that while certain flow features are not resolved by the lower-mode Time-Spectral cases, Fig. 6 suggests that using $N \geq 9$ matches the time-accurate drag-coefficient for nearly the entire period of oscillation. Vorticity magnitude varies from 0 in white to greater than or equal to 1 in black. 


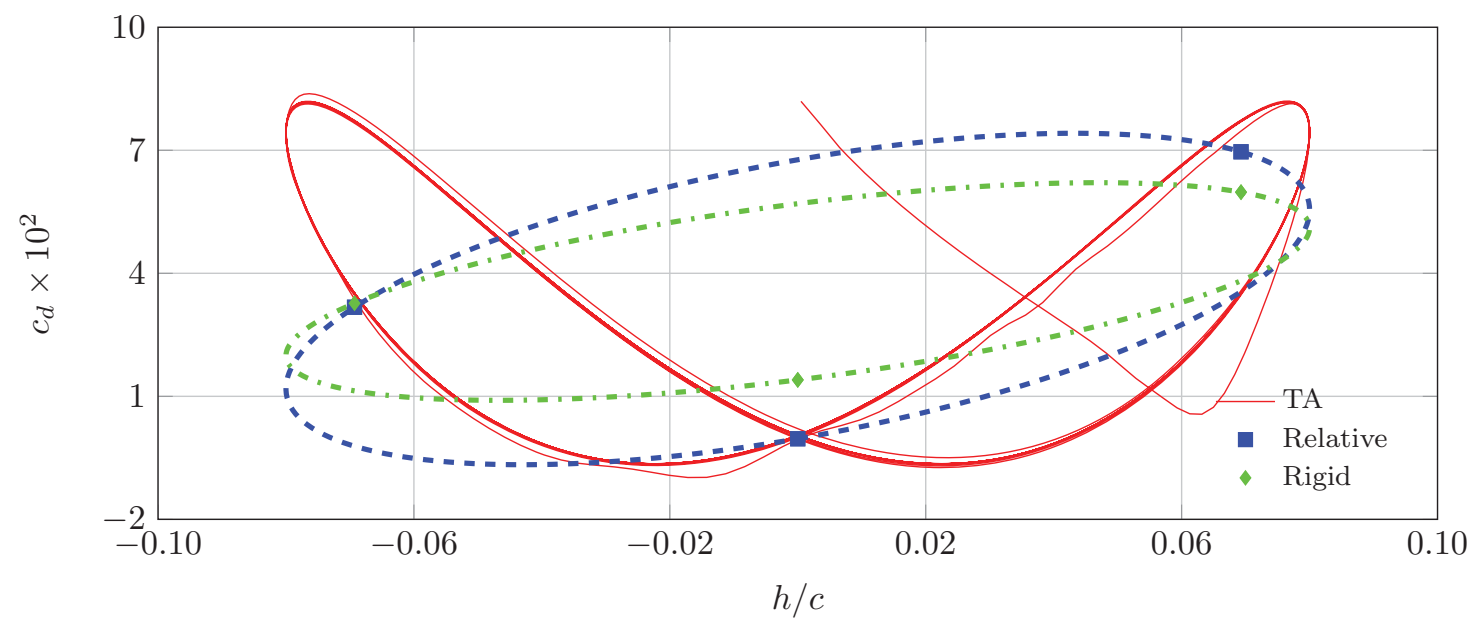

(a) $N=3$

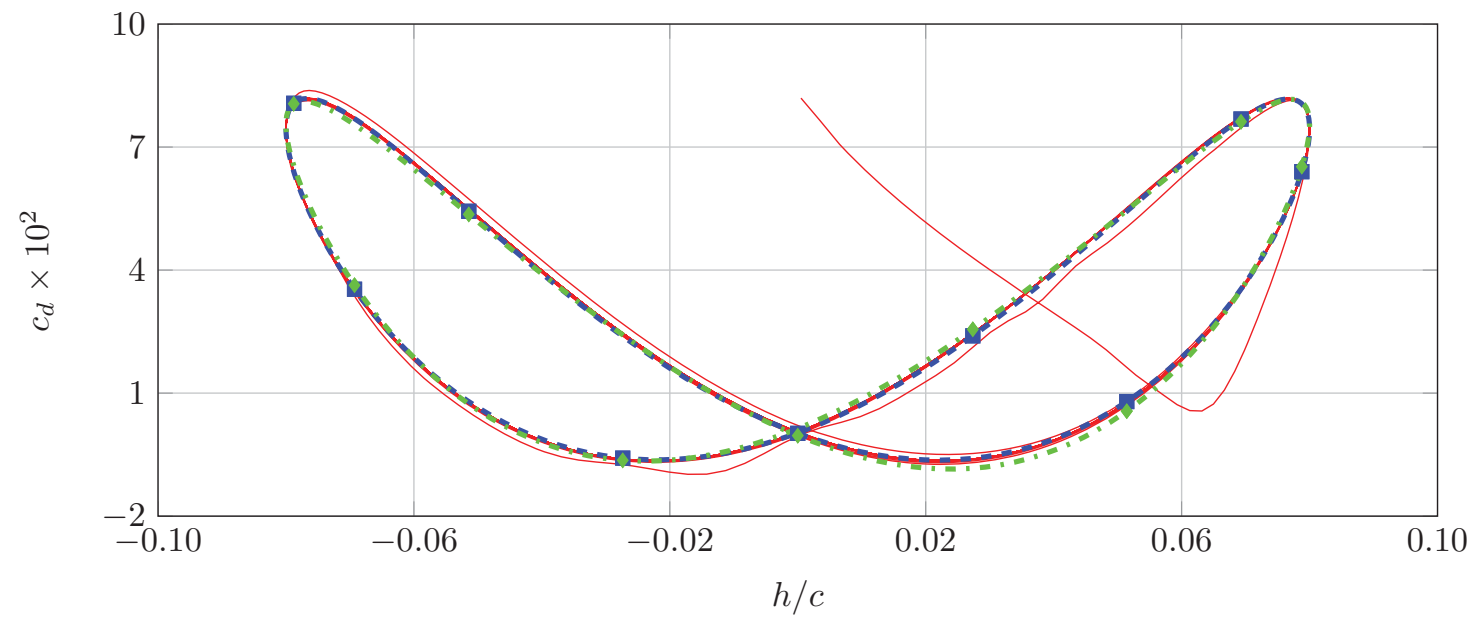

(b) $N=9$

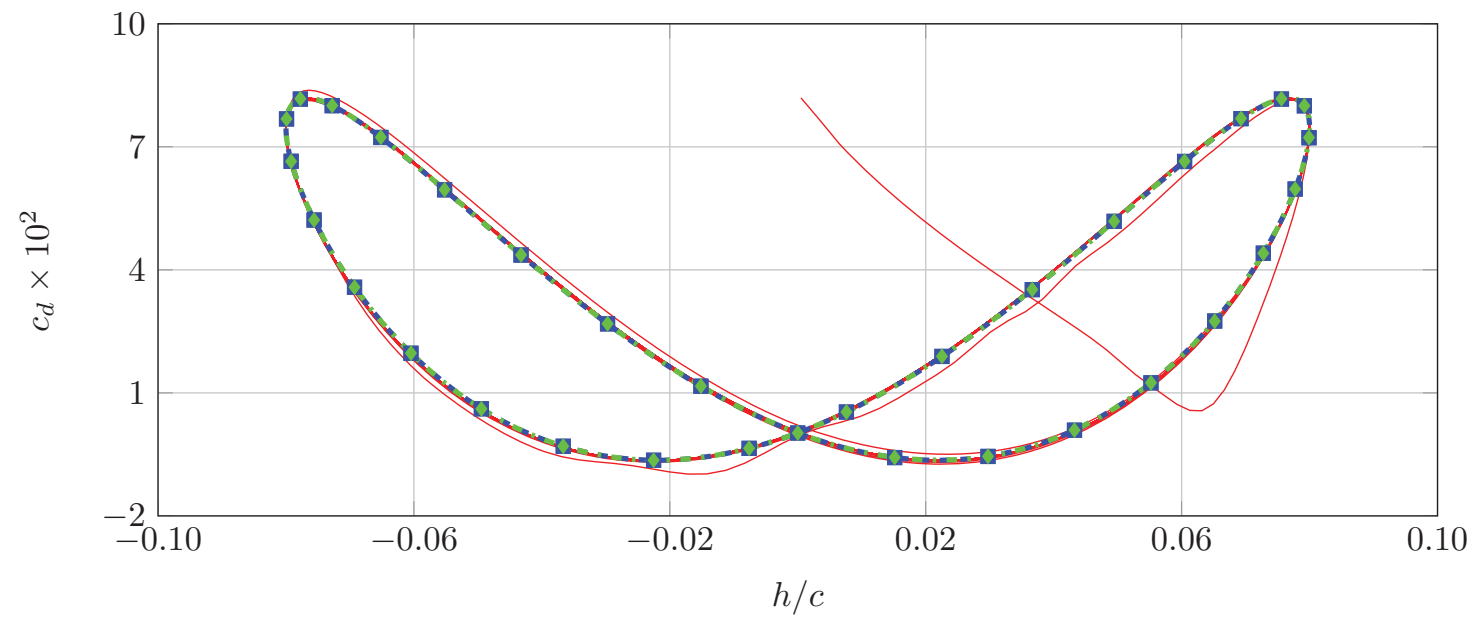

(c) $N=33$

Figure 5: Laminar Plunging NACA 0012 Airfoil. Drag-producing case at $M_{\infty}=0.2$ and $R e=1850$. Time Spectral versus time accurate drag coefficients for $N \in\{3,9,33\}$. Ten periods of the rigid-motion time-accurate solution are plotted in red from steady-state startup. Blue squares locate the drag coefficient values at the Time-Spectral collocation points for relative-body motion. Relative-body drag coefficients computed from an interpolation of the Time-Spectral solution to 201 points shown with the blue-hashed line. Green diamonds locate the drag coefficient values at the Time-Spectral collocation points for rigid-body motion. Rigid-body drag coefficients computed from an interpolation of the Time-Spectral solution to 201 points shown with the green-hashed line. 


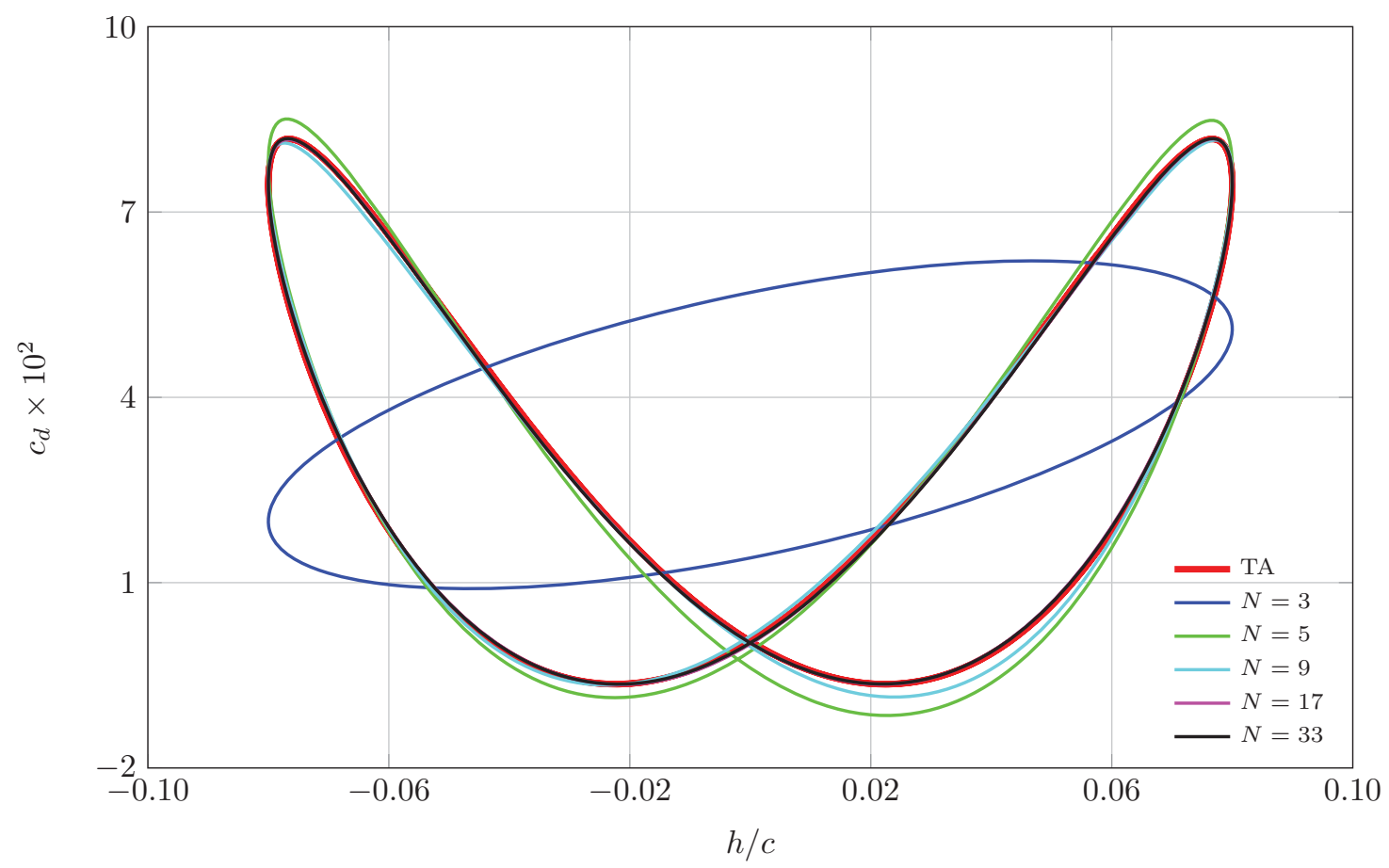

(a) Rigid Motion

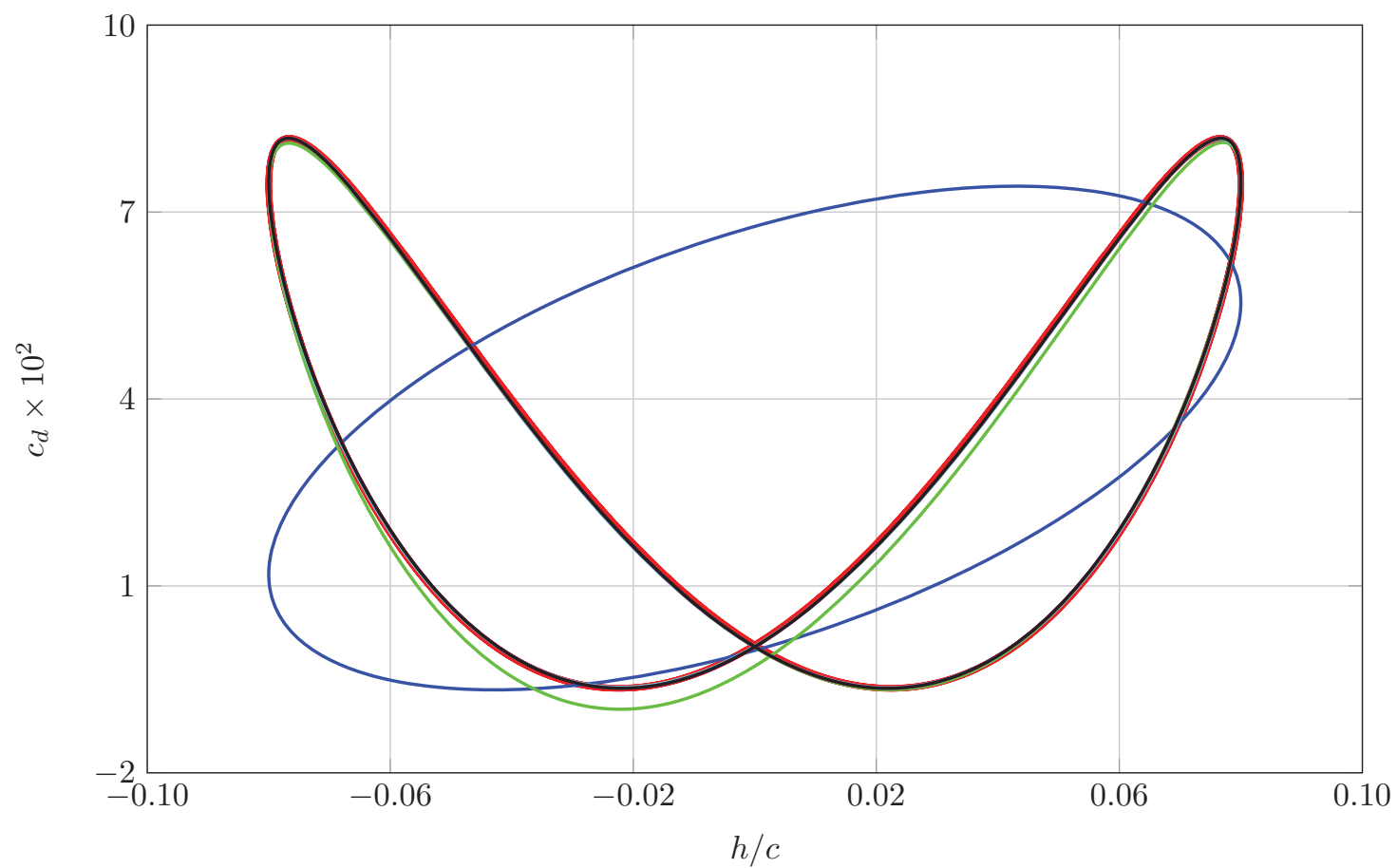

(b) Relative Motion

Figure 6: Laminar Plunging NACA 0012 Airfoil. Drag-producing case at $M_{\infty}=0.2$ and $R e=1850$. Convergence of (a) rigid- and (b) relative- motion Time-Spectral drag coefficients to the periodic steadystate time-accurate calculation for $N \in\{3,5,9,17,33\}$. 


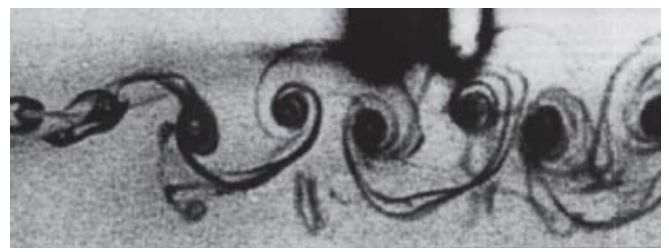

(a) Experimental [35]

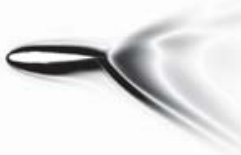

(c) Rigid, $N=3$

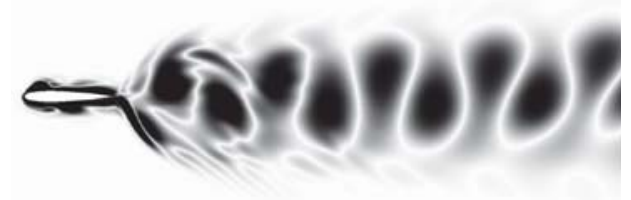

(e) Rigid, $N=5$

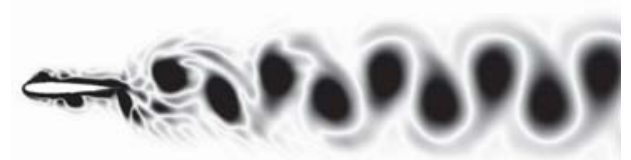

(g) Rigid, $N=9$

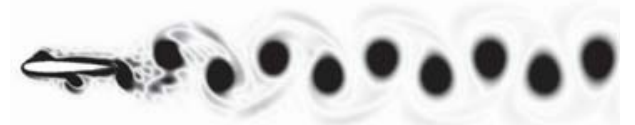

(i) Rigid, $N=17$

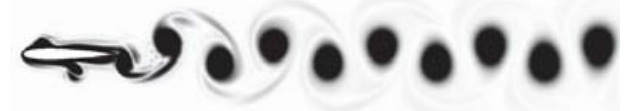

(k) Rigid, $N=33$

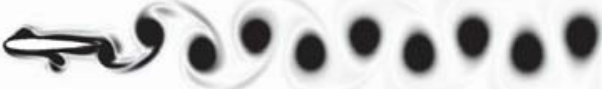

(b) Time Accurate

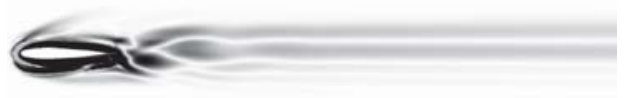

(d) Relative, $N=3$

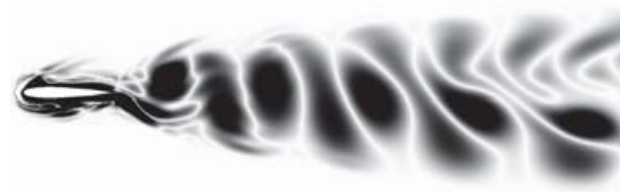

(f) Relative, $N=5$

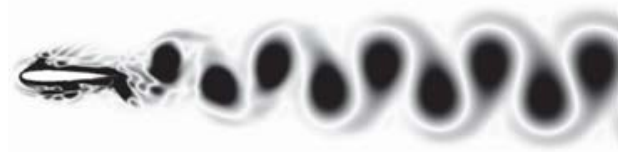

(h) Relative, $N=9$

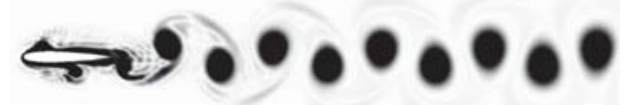

(j) Relative, $N=17$

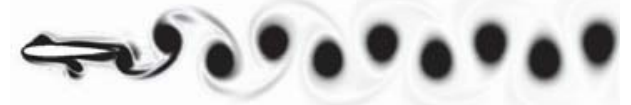

(1) Relative, $N=33$

Figure 7: Laminar Plunging NACA 0012 Airfoil. Thrust-producing case at $M_{\infty}=0.2$ and $R e=1850$. Visualization of vorticity magnitude. Comparison of experimental with computed time-accurate and Time-Spectral results using $N \in\{3,5,9,17,33\}$ for both rigid- and relative-body motion. 


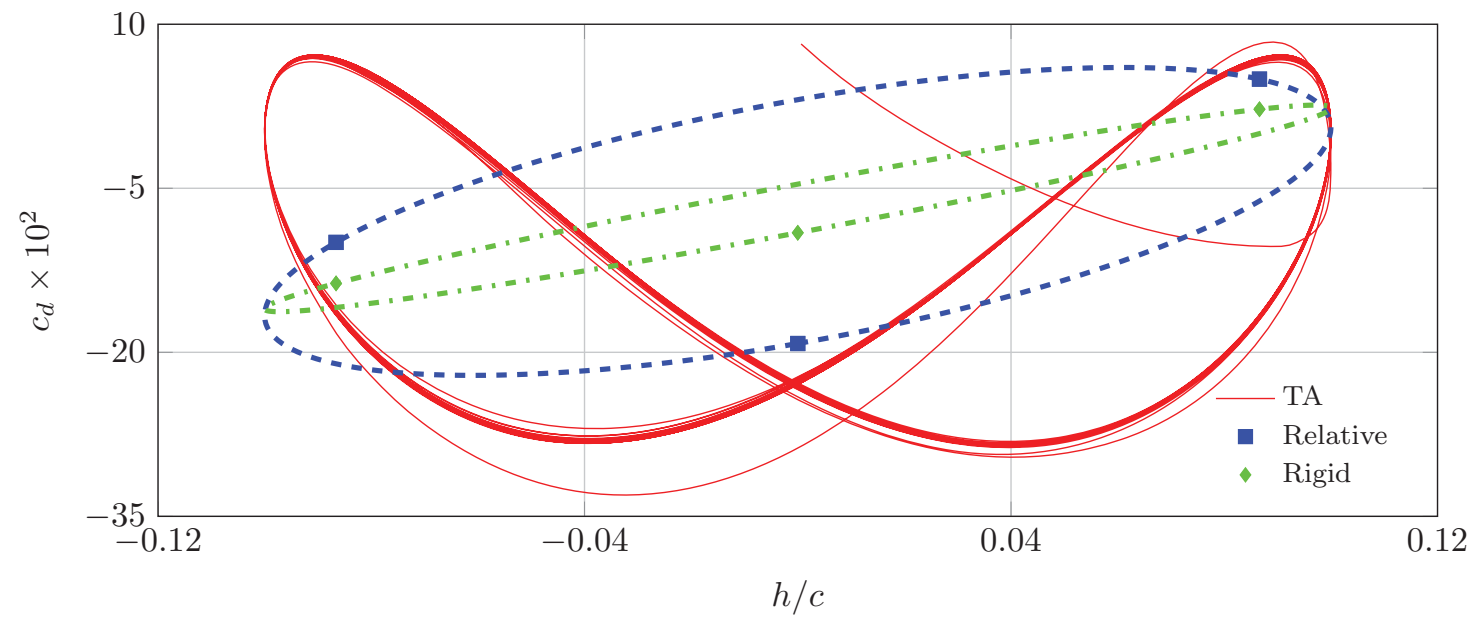

(a) $N=3$

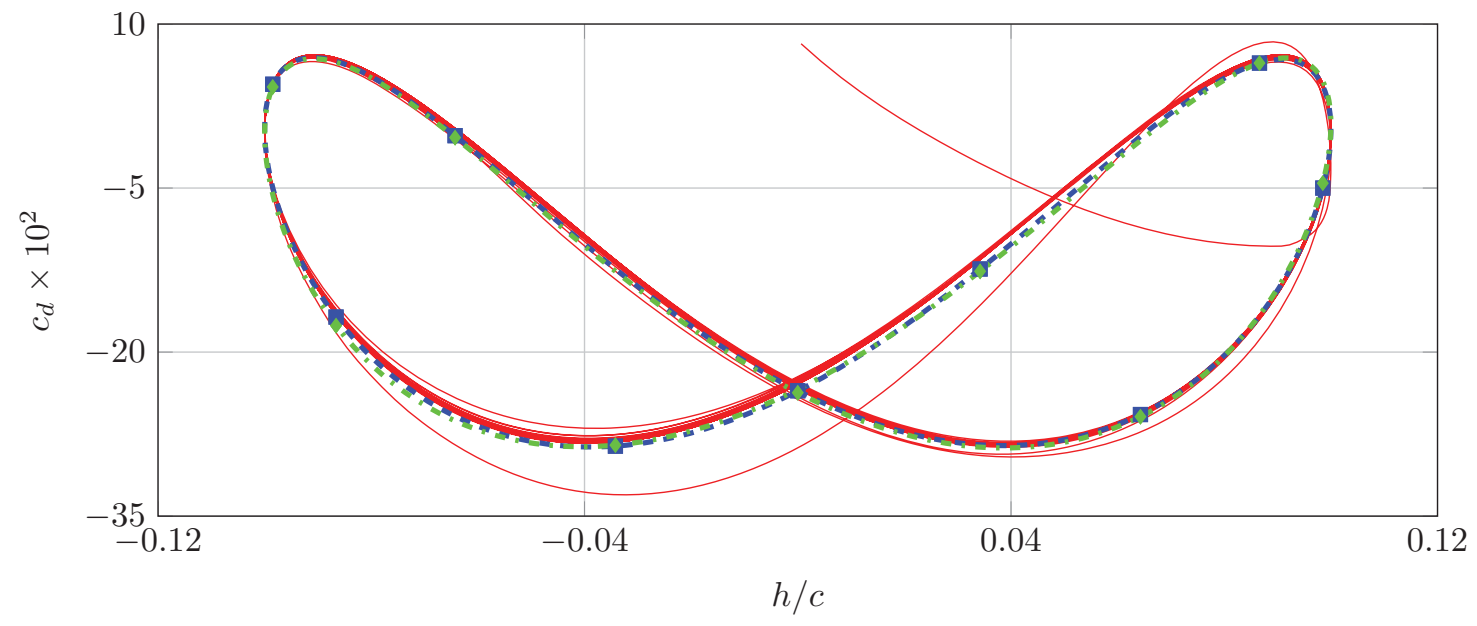

(b) $N=9$

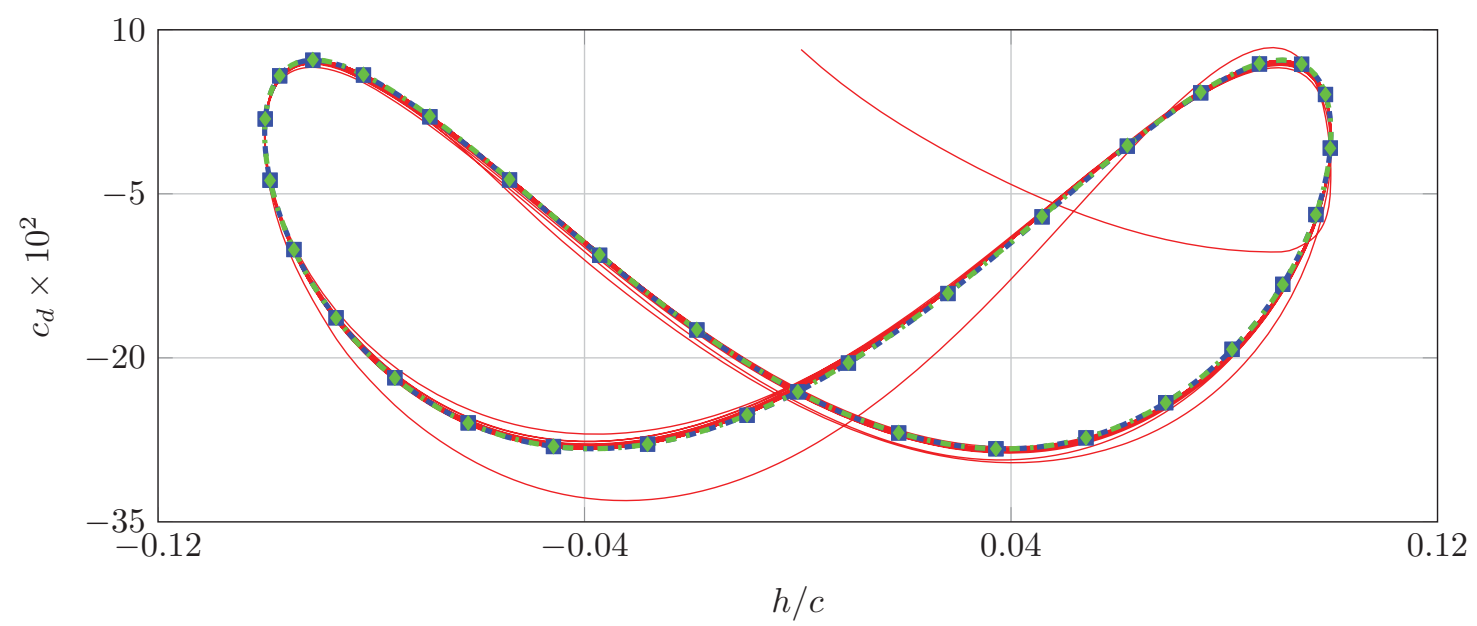

(c) $N=33$

Figure 8: Laminar Plunging NACA 0012 Airfoil. Thrust-producing case at $M_{\infty}=0.2$ and $R e=1850$. Time Spectral versus time accurate drag coefficients for $N \in\{3,9,33\}$. The time-accurate solution is plotted in red from steadystate startup. Blue squares locate the drag coefficient values at the Time-Spectral collocation points for relative-body motion. Relative-body drag coefficients computed from an interpolation of the Time-Spectral solution to 201 points shown with the blue-hashed line. Green diamonds locate the drag coefficient values at the Time-Spectral collocation points for rigid-body motion. Rigid-body drag coefficients computed from an interpolation of the Time-Spectral solution to 201 points shown with the green-hashed line. 


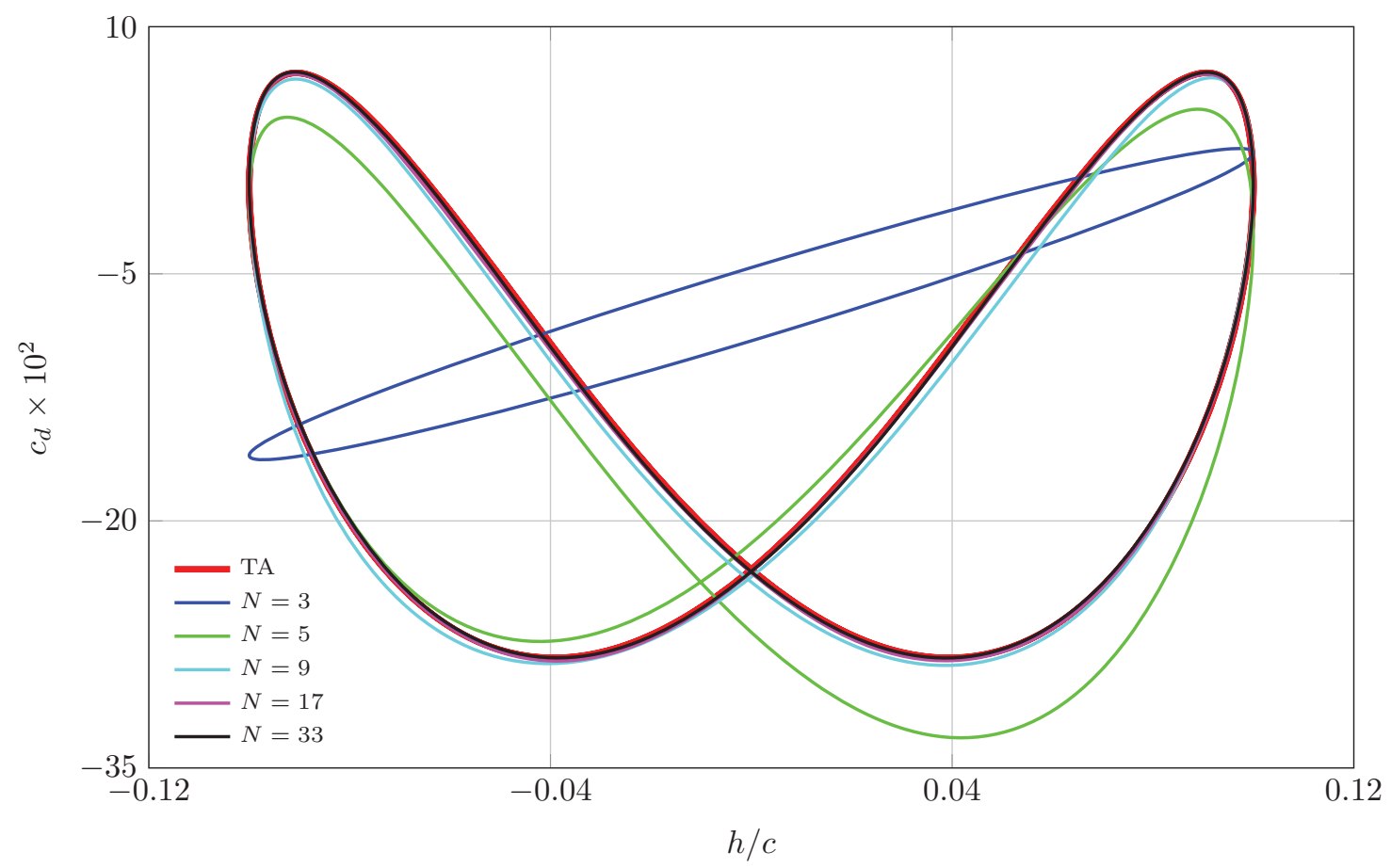

(a) Rigid Motion

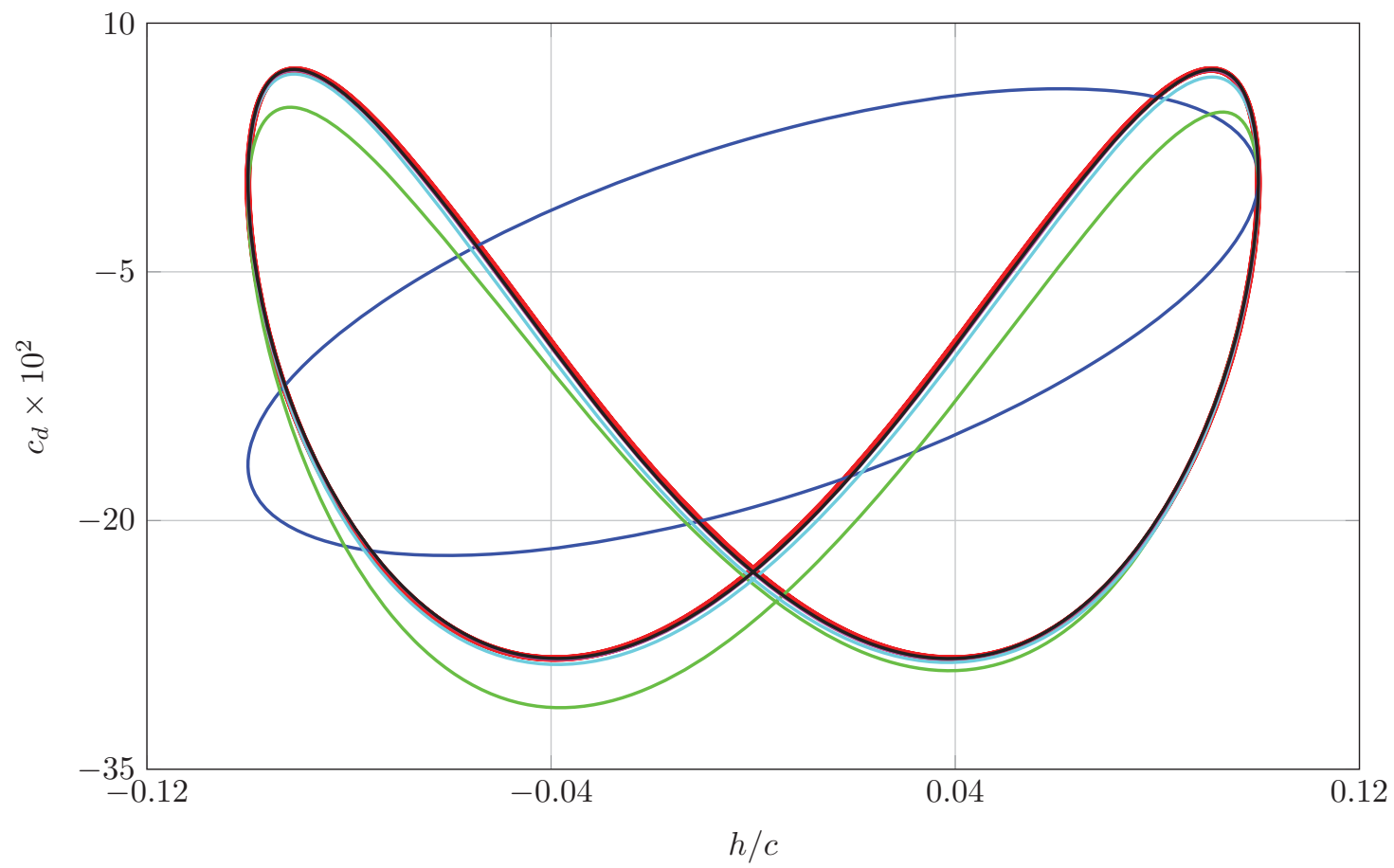

(b) Relative Motion

Figure 9: Laminar Plunging NACA 0012 Airfoil. Thrust-producing case at $M_{\infty}=0.2$ and $R e=1850$. Convergence of (a) rigid- and (b) relative-motion Time-Spectral drag coefficients to the periodic steady-state time-accurate calculation for $N \in\{3,5,9,17,33\}$. 


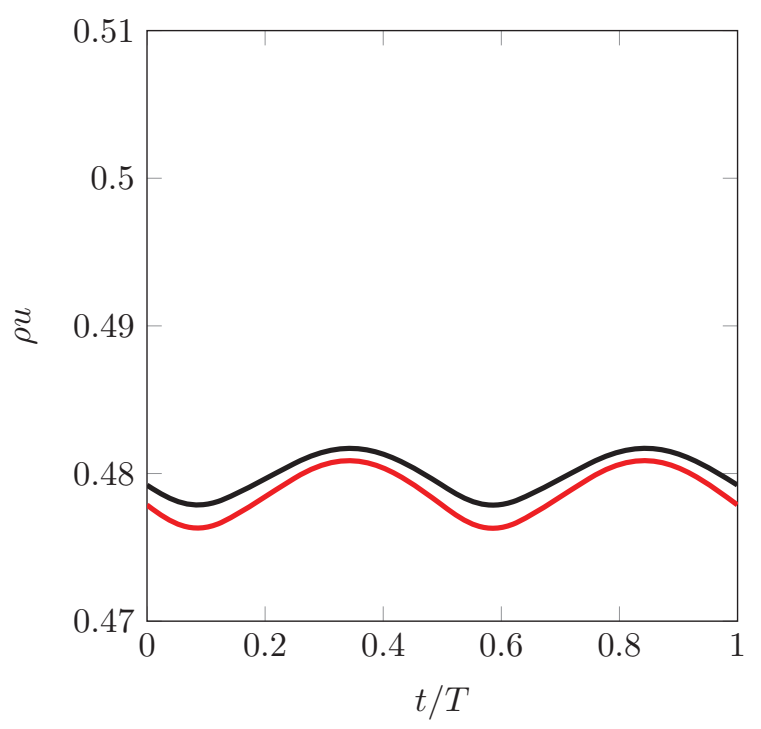

(a) Streamwise momentum, $\rho u$, over one period.

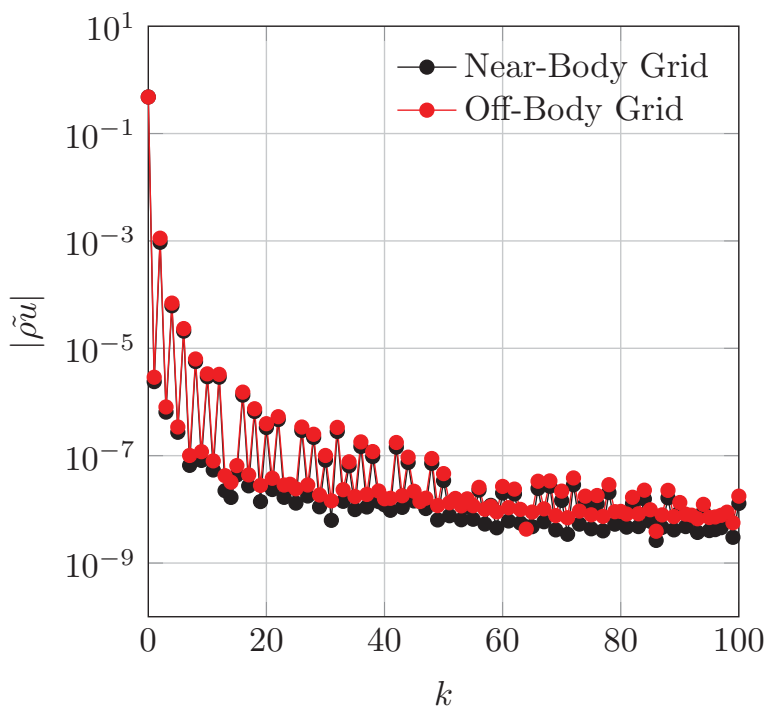

(b) Spectrum of streamwise momentum, $|\tilde{\rho u}|$.

Figure 10: Plunging NACA 0012 Airfoil. Inviscid case with $h=0.5$ and $M_{\infty}=0.5$. Time and frequency response of subsonic plunging airfoil with an off-body grid translating with the airfoil. The frequency components at the near- and off-body nodes are nearly identical.

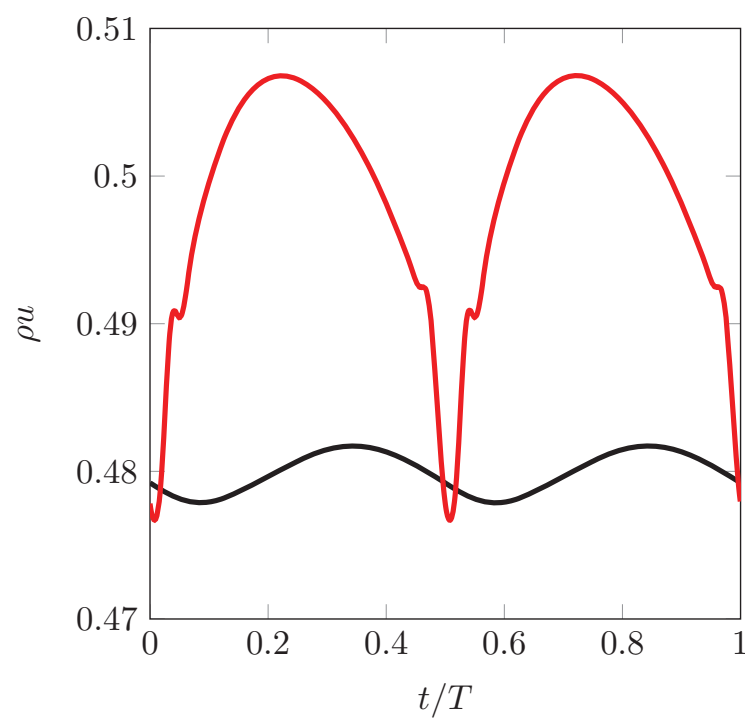

(a) Streamwise momentum, $\rho u$, over one period.

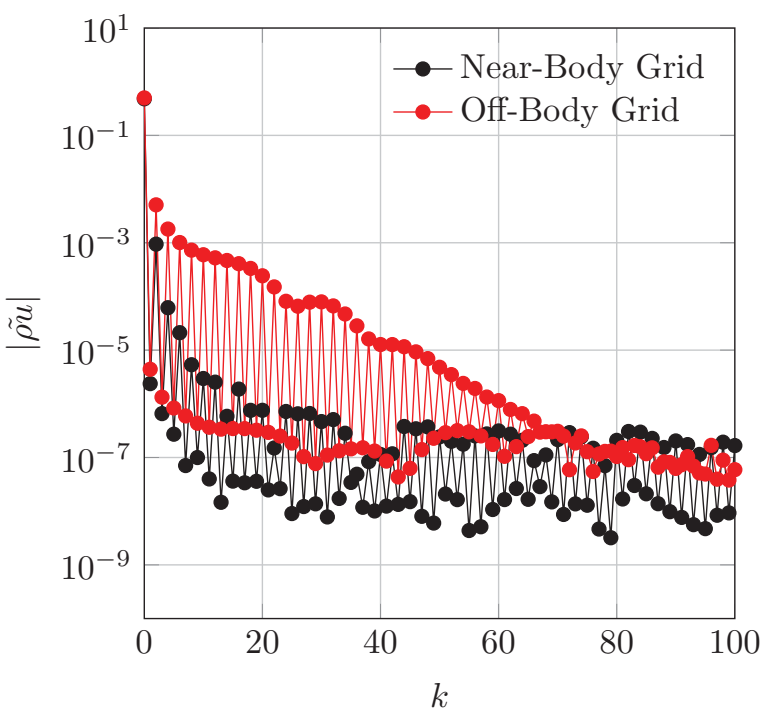

(b) Spectrum of streamwise momentum, $|\tilde{\rho u}|$.

Figure 11: Plunging NACA 0012 Airfoil. Inviscid case with $h=0.5$ and $M_{\infty}=0.5$. Time and frequency response of subsonic plunging airfoil with a stationary background grid. The frequency components at the near- and off-body nodes are strikingly different with many more modes required to resolve the solution at the node on the stationary background grid. The node on the background grid is the same one described in Fig. 10a, but the background grid was translating with the airfoil in that case and only a few modes are required to resolve the solution. 


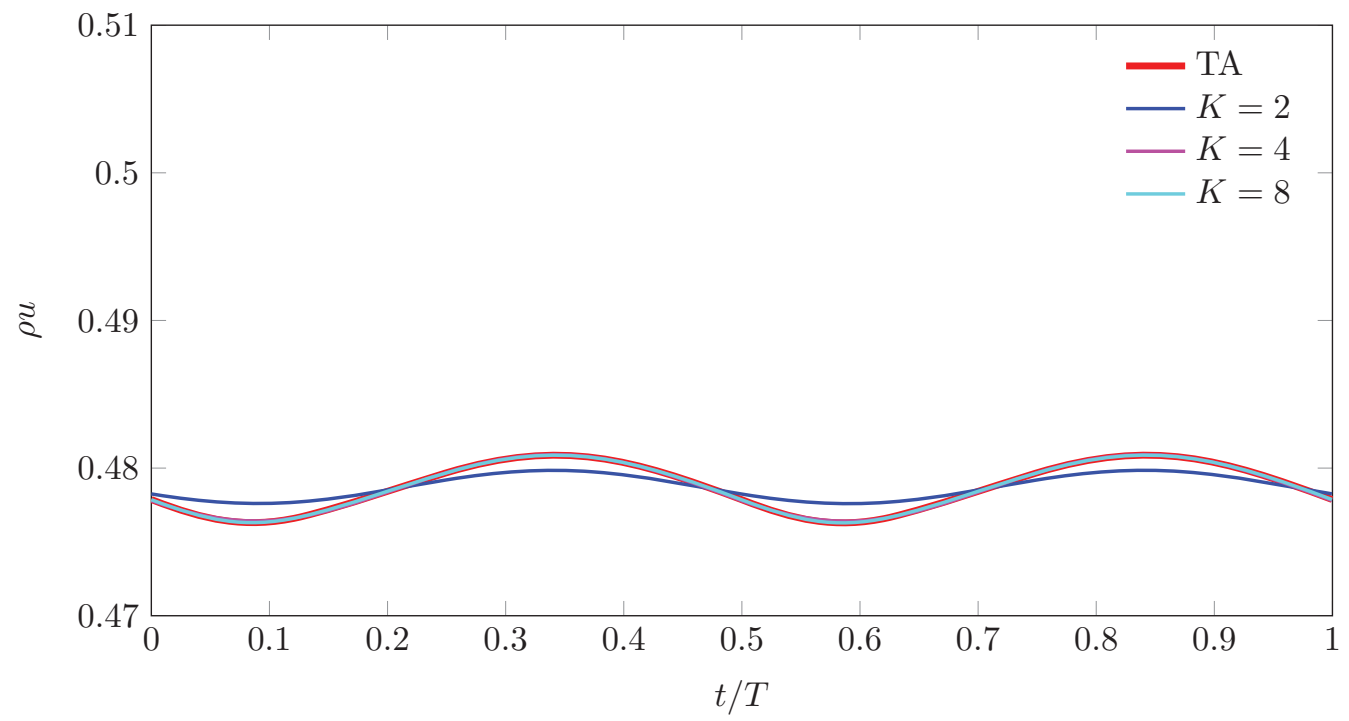

(a) Translating node on background grid in rigid-body motion.

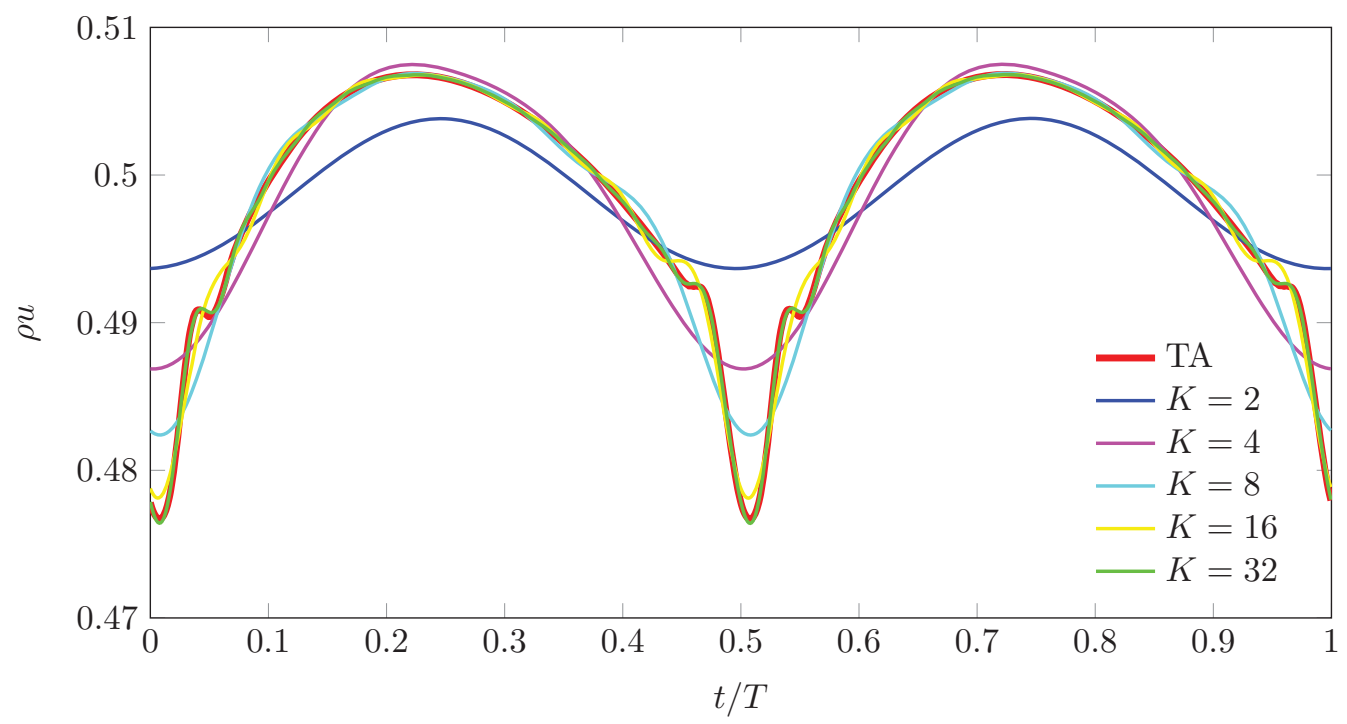

(b) Stationary node on background grid in relative-body motion

Figure 12: Plunging NACA 0012 Airfoil. Inviscid case with $h=0.5$ and $M_{\infty}=0.5$. Temporal reconstruction of streamwise momentum, $\rho u$, at a node on the off-body grid (a) translating with the airfoil and (b) stationary relative to the translating airfoil. The solution at the stationary node requires approximately $K=32$ modes to reasonably reconstruct the continuous signal whereas the solution at the translating node requires approximately $K=4$ modes, suggesting an inherent inefficiency of employing relative motion. However, rigid-body motion is not universally applicable and therefore relative-body motion must be used for certain configurations. 


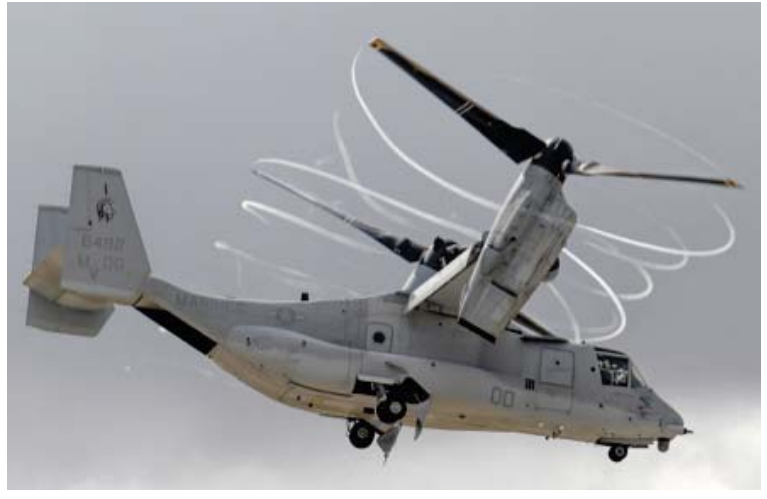

(a) Full Configuration

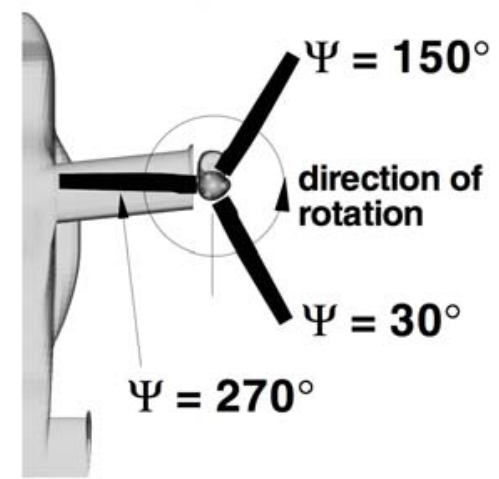

(b) Azimuthal angle, $\Psi$

Figure 13: V-22 Osprey (a) full configuration [44] (b) definition of azimuthal angle, $\Psi$, taken from [41].

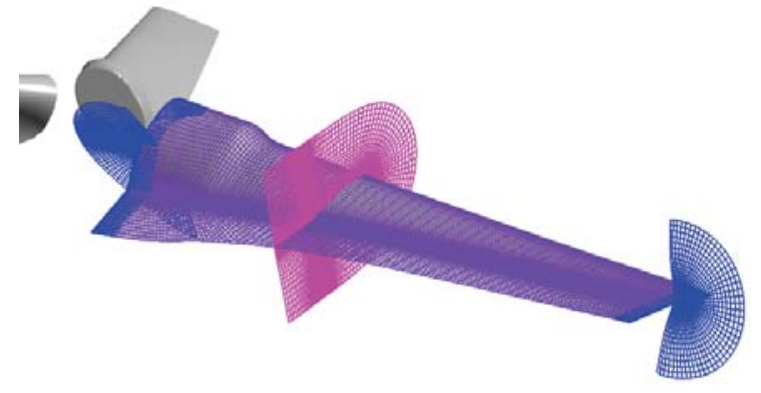

(a) Blades with near-body grids.

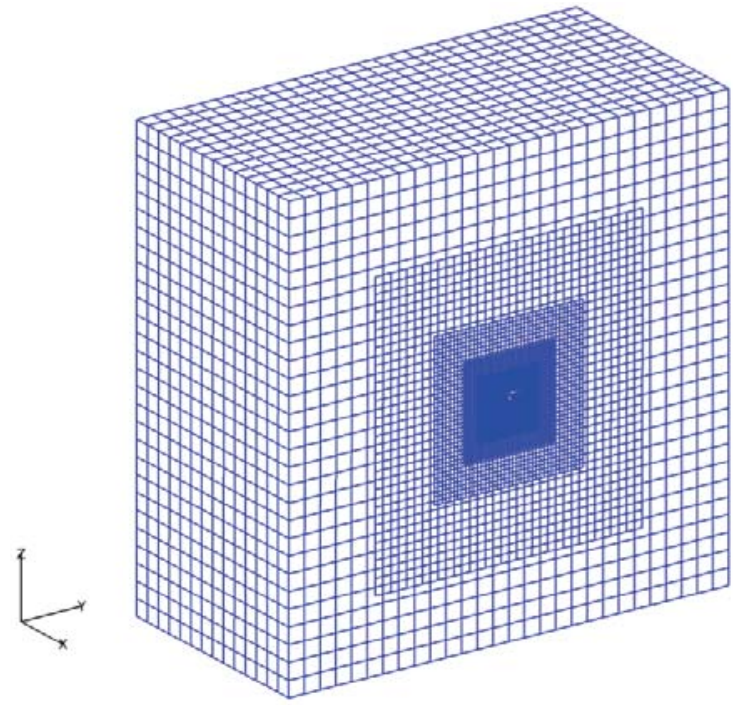

(b) Off-body grid-system.

Figure 14: Isolated V-22 Osprey Tiltrotor in Hover. Geometry and grid system. Each blade is wrapped in an C-mesh with tip and root cap grids and the rotor is embedded in a hierarchy of isotropic Cartesian off-body grids. The domain of the off-body system is cut away to reveal the structure of the isotropic Cartesian grids. 


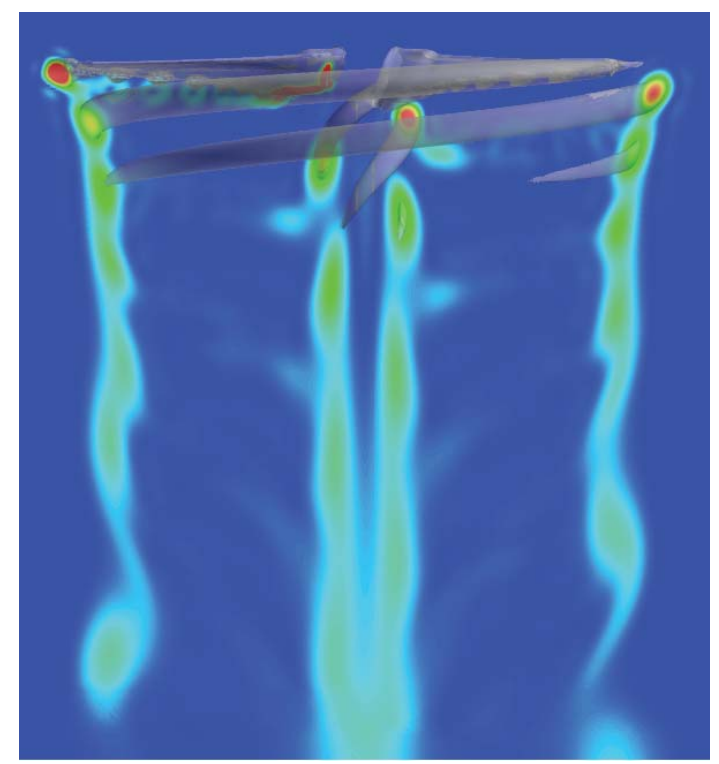

(a) Time Accurate

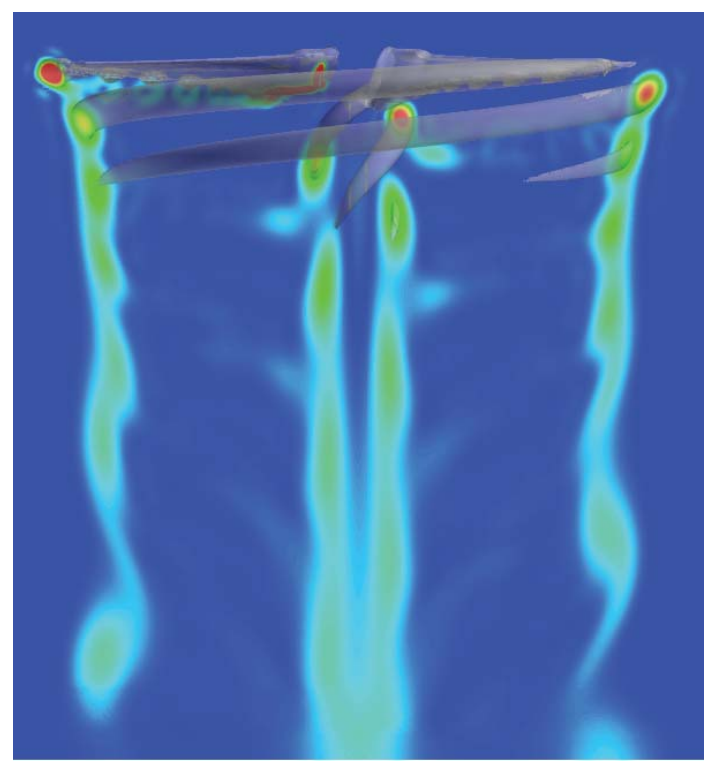

(b) Time Spectral

Figure 15: Isolated V-22 Osprey Tiltrotor in Hover. Comparison of iso-contours of vorticity for time-accurate and Time-Spectral simulations. Contours of vorticity magnitude are plotted in a constant $y$-plane, ranging from a value of zero in blue to 0.01 in red.

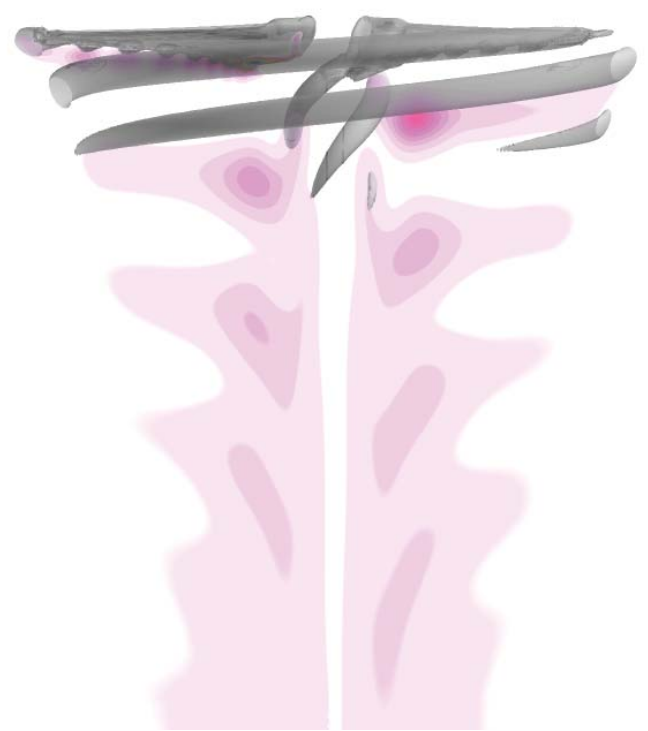

(a) Time Accurate

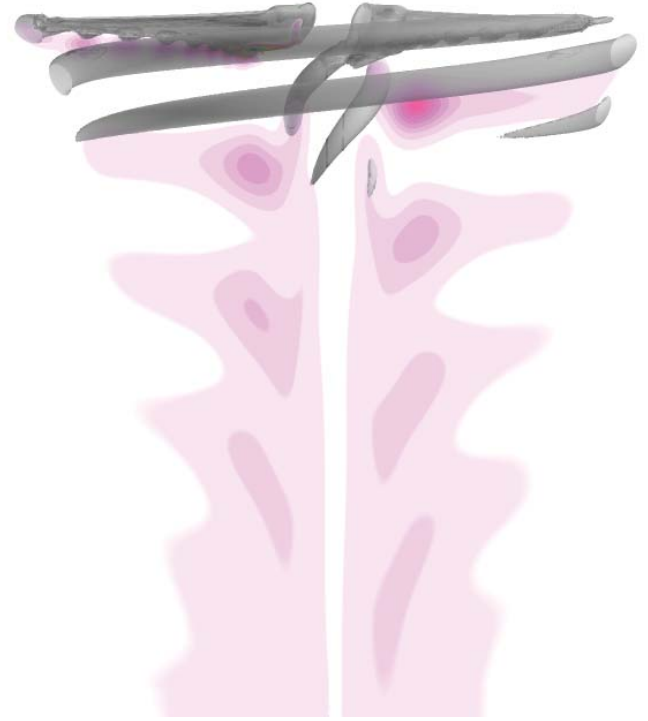

(b) Time Spectral

Figure 16: Isolated V-22 Osprey Tiltrotor in Hover. Comparison of iso-contours of vorticity for time-accurate and Time-Spectral simulations. Contours of undamped turbulent eddy viscosity, $\tilde{\nu}$, are plotted in a constant $y$-plane, ranging from a value of zero in white to 900 in black. 


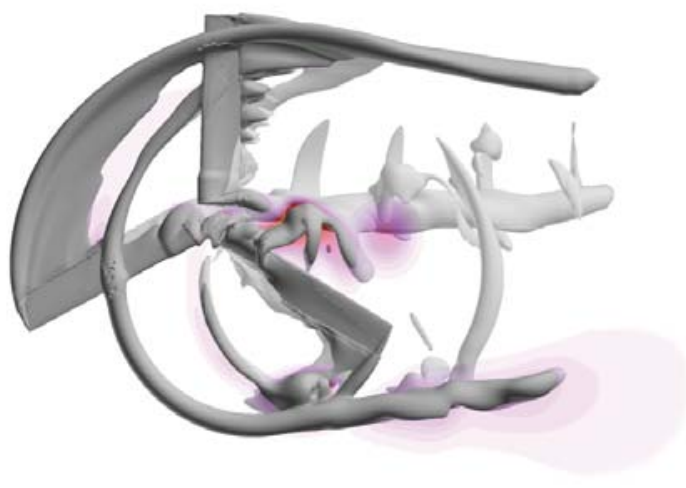

(a) Time Accurate, $N=1440$

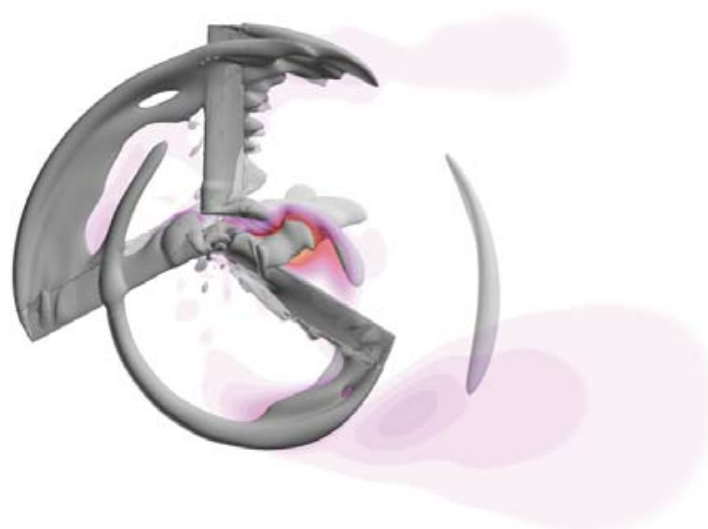

(c) Time Spectral, $N=21$

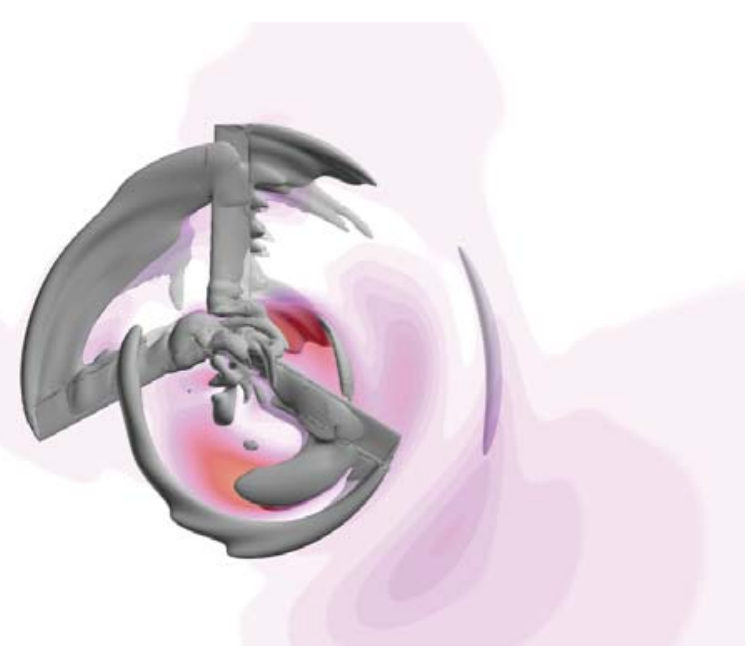

(b) Time Spectral, $N=11$

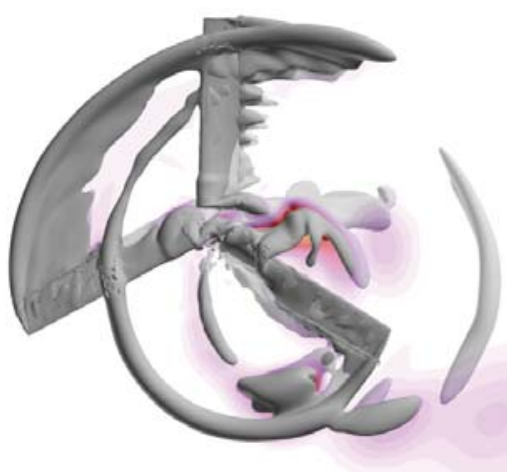

(d) Time Spectral, $N=31$

Figure 17: Isolated V-22 Osprey Tiltrotor in Forward Flight. Top view of iso-contours of vorticity for timeaccurate and Time-Spectral calculations with advance ratio $\mu=0.2$ and tip Mach number $M_{\text {tip }}=0.625$. Time-Spectral simulations using $N \in\{11,21,31\}$ are presented to demonstrate the convergence towards the time-accurate result with the addition of temporal modes. Contours of undamped eddy viscosity are plotted in a constant $z$ plane. 


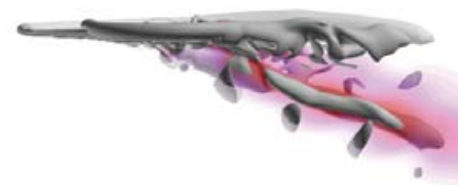

(a) Time Accurate, $N=1440$

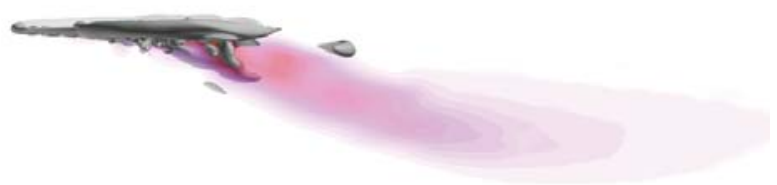

(c) Time Spectral, $N=21$

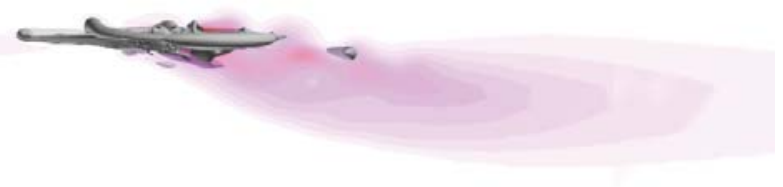

(b) Time Spectral, $N=11$

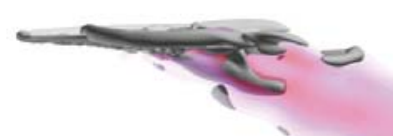

Figure 18: Isolated V-22 Osprey Tiltrotor in Forward Flight. Side view of iso-contours of vorticity for timeaccurate and Time-Spectral calculations with advance ratio $\mu=0.2$ and tip Mach number $M_{\text {tip }}=0.625$. Time-Spectral simulations using $N \in\{11,21,31\}$ are presented to demonstrate the convergence towards the time-accurate result with the addition of temporal modes. Contours of undamped eddy viscosity are plotted in a constant $y$ plane. 


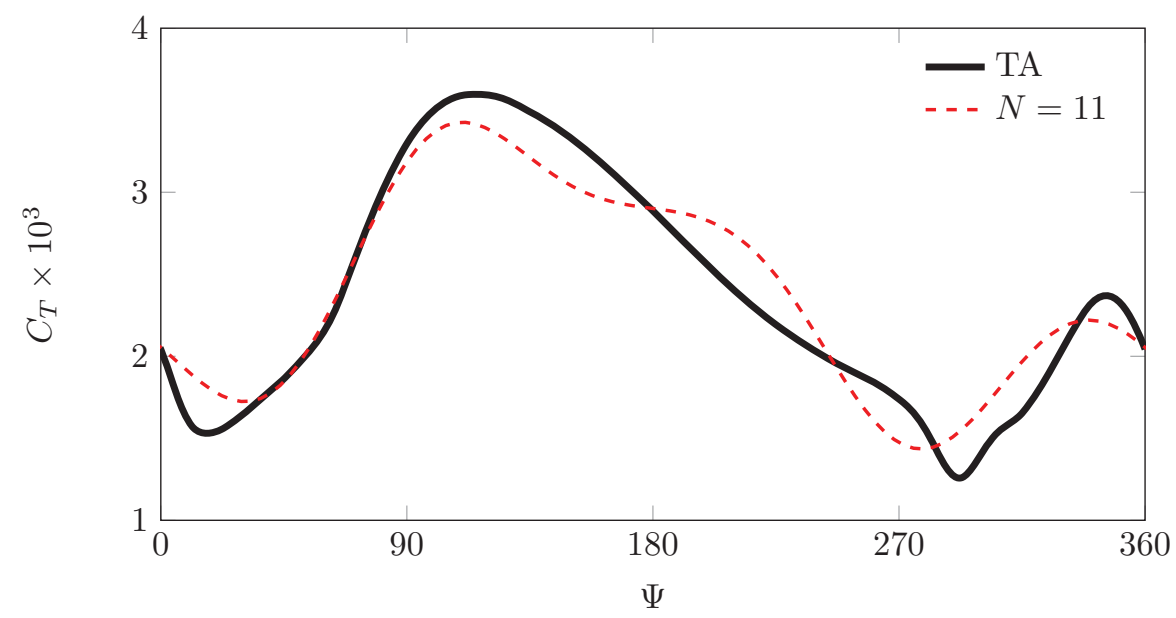

(a) Five harmonics, $N=11$

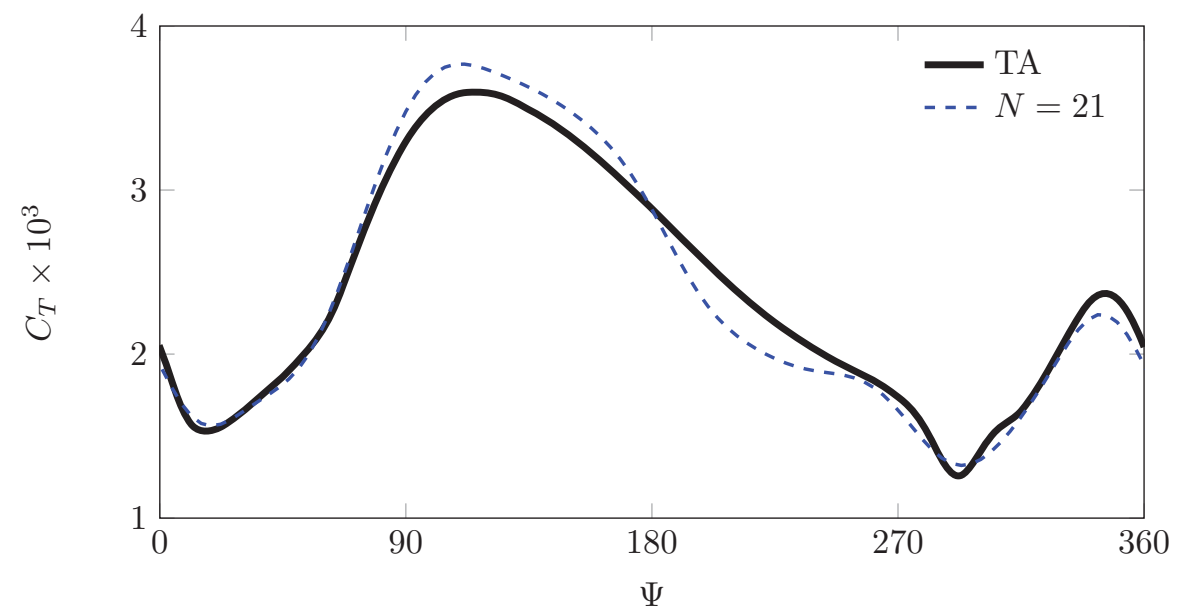

(b) Ten Harmonics, $N=21$

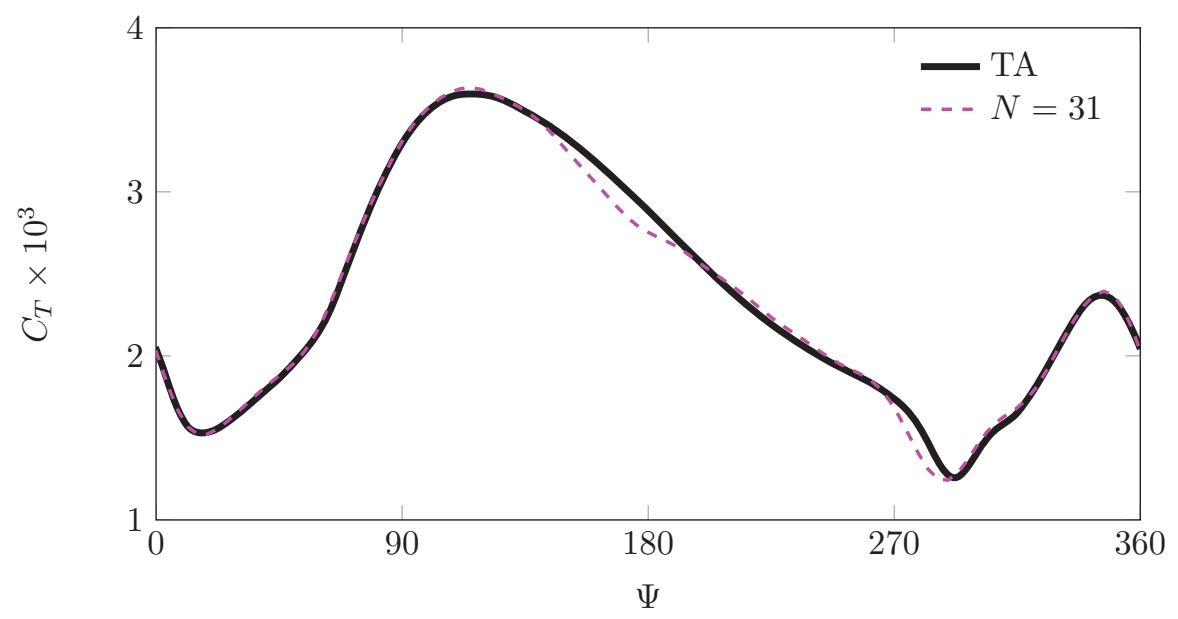

(c) Fifteen harmonics, $N=31$

Figure 19: Isolated V-22 Osprey Tiltrotor in Forward Flight. Reconstruction of Time-Spectral thrust coefficient, $C_{T}$, for $N \in\{11,21,31\}$ against the time-accurate calculation versus $\Psi$ using a time step of equivalent to $0.25^{\circ}, \Delta t=T / 1440$. 